\title{
COUNTING DIVISORS WITH PRESCRIBED SINGULARITIES BY
}

\author{
ISRAEL VAINSENCHER
}

\begin{abstract}
Given a family of divisors $\left\{D_{s}\right\}$ in a family of smooth varieties $\left\{Y_{s}\right\}$ and a sequence of integers $m_{1}, \ldots, m_{t}$, we study the scheme parametrizing the points $\left(s, y_{1}, \ldots, y_{t}\right)$ such that $y_{i}$ is a (possibly infinitely near) $m_{i}$-fold point of $D_{s}$. We obtain a general formula which yields, as special cases, the formula of de Jonquières and other classical results of Enumerative Geometry. We also study the questions of finiteness and the multiplicities of the solutions.
\end{abstract}

1. Introduction. The aim of this work is to obtain formulas for the number of divisors in a family which possess points with specified multiplicities. For instance, if the family is given by the hyperplane sections of a smooth subvariety $Y$ of a projective space, we wish to count the hyperplanes satisfying specified contact conditions with $Y$.

About a century ago de Jonquières published his Mémoire [10], exhibiting a formula for the number of plane curves of a given degree having prescribed contacts with a fixed plane curve. The formula itself has generated much interest (cf. Enriques and Chisini [3], MacDonald [16], [17] and Mattuck [19]). Shortly afterwards, the flourishing school of Enumerative Geometry produced a wealth of results on contacts of lines and planes with surfaces in 3-space and other enumerative problems (cf. Zeuthen and Pieri [27]).

The next generation of algebraic geometers somewhat changed the emphasis, from the counting of singularities per se, to the discovery of certain invariants definable in terms of those singularities. Thus, one finds in C. Segre [24, p. 75] the definition of the genus of a curve in terms of the invariant $\nu-2 n$, where $\nu$ is the number of double points of a $g_{n}^{1}$ on the curve. The Zeuthen-Segre invariant (Enriques [4, p. 167]) and the very definition of the canonical system [4, p. 49] are further examples of the "new" trend.

Possessing now as we do, a well-developed intersection theory, it is natural to try and go back to the origins, and vindicate (to today's taste and sense of rigor) those classical formulas. In fact, Hilbert's 15 th problem calls for “... the actual carrying out of the process of elimination in the case of equations of special form in such a way that the degree of the final equations and the multiplicity of their solutions may be foreseen" [8, p. 464]. We hope this work will provide a step in this direction. For a survey on recent developments on the subject, we refer to Kleiman [12], [13].

Received by the editors May 11, 1979 and, in revised form, July 17, 1980.

AMS (MOS) subject classifications (1970). Primary 14N10, 14C15, $14 \mathrm{~F} 05$.

Key words and phrases. Projective algebraic variety, families of divisors, multiple points, scheme of zeros, locally free sheaves, Chern classes, contact conditions. 
The paper is organized as follows. In order to study singular divisors in a family $\left\{D_{s}\right\}$, it is convenient to look at the pairs $(y, s)$ such that $y$ is a singular point of $D_{s}$. This leads to our definition of incidence correspondences in \$2. The case where the ambient spaces $Y_{s}$ are curves is treated in $\$ 3$. Here is also handled the case of arbitrary dimension but with just one assigned multiplicity. In $\$ 4$ we apply the machinery developed so far to retrieve the formula for the degree of the dual variety (4.2), the Zeuthen-Segre invariant (4.3) and the formula of de Jonquières (4.4). In $\$ 5$ we derive formulas for contacts of lines with a surface in 3-space. In $\S \S 6$ and 7 we introduce a step-by-step procedure to handle the case of arbitrary relative dimension. Roughly, to get a hold on the divisors that possess, say, one triple and another double point, we first restrict to the subfamily of divisors with a triple point. Then we construct a new family by taking (virtual) transforms at each assigned triple point, and finally we search for the double points in the new family. In $\$ 8$ we obtain formulas that apply to the counting of bitangent and tritangent planes of a surface in 3-space. We explain how to deal with contacts of a hypersurface with higher-dimensional linear spaces and give an example in 4-space (8.4). We also show how to get formulas for the number of curves (embedded in a family of surfaces) displaying specified coincidences of tangents at a singularity. This yields, as special cases, the number of cusps of a general net on a surface (8.6.3), as well as the cuspnodes of a general web (8.6.4). The last two paragraphs are devoted to the questions of finiteness and multiplicity of the solutions.

As a general proviso, all schemes are assumed to be quasiprojective over an algebraically closed field $k$, and points are $k$-rational. The intersection theory used here is that of Fulton [5]. If $X$ is a scheme and $Z \subseteq X$ is closed, we denote by [Z] the class of $Z$ in the Chow group $A$. $(X)$. We denote by $\int z$ the degree of a zero cycle $z$ of a projective scheme.

ACKnowledgement. This work is based on my Ph. D. thesis (MIT, 1976). Many thanks are due to S. L. Kleiman, my adviser, for all his encouragement and helpful discussions.

2. Incidence correspondences. Suppose we are given a diagram of maps of schemes,

$$
\begin{gathered}
\underline{W} \subset \frac{X}{\downarrow} \supset \underline{D} \\
\underline{S}
\end{gathered}
$$

We think of $\underline{X} \rightarrow \underline{S}$ as a family of ambient spaces, and of $\underline{W}$ and $\underline{D}$ as the total spaces of families of subschemes $\left\{\underline{W}_{s}\right\}_{s \in \underline{S}},\left\{\underline{D}_{s}\right\}_{s \in \underline{s}}$. We call a subscheme of $\underline{S}$ the incidence correspondence of $\underline{W}$ in $\underline{D}$ if it has the following universal property: a map $T \rightarrow \underline{S}$ factors through it iff $\underline{W}_{T} \subset \underline{D}_{T}$ holds (as subschemes of the pullback $\left.\underline{X}_{T}=\underline{X}_{S} \times T\right)$.

Proposition (2.2) will give a criterion for the existence of incidence correspondences. It is instructive, however, to look first at some of the examples that motivate the whole business.

(2.1) ExAmples. (1) Let $Y$ be a smooth, projective variety. Let $\Delta \subset Y \times Y$ be the diagonal subvariety. Given a positive integer $m$, let $m \Delta$ denote the subscheme of 
$Y \times Y$ with ideal $I(m \Delta)=I(\Delta)^{m}$. Let $S$ be (the parameter space of) a linear system on $Y$ with universal divisor $D \subset Y \times S$. Put $\underline{S}=Y \times S, \underline{X}=Y \times \underline{S}$ and take $\underline{W}$ and $\underline{D}$ to be the pullbacks of $m \Delta$ and $D$ to $\underline{X}$. Then the incidence correspondence of $\underline{W}$ in $\underline{D}$ consists of the pairs $(y, s)$ such that $y$ is an (at least) $m$-fold point of $D_{s}$.

(2) Suppose now $\operatorname{dim} Y=1$. Put $Y^{t}=Y \times \cdots \times Y$ (t-times). Let $\Delta_{0 i}$ denote the diagonal $\left\{\left(y_{0}, \ldots, y_{t}\right) \in Y \times\left. Y^{t}\right|_{y_{0}}=y_{i}\right\}$. Given a sequence of positive integers $\underline{m}:=m_{1}, \ldots, m_{t}$, let $\underline{m} \Delta$ stand for the positive divisor

$$
\underline{m} \Delta=m_{1} \Delta_{01}+\cdots+m_{t} \Delta_{0 t} .
$$

Observe that the fibre of $\underline{m} \Delta$ over $\left(y_{1}, \ldots, y_{t}\right) \in Y^{t}$ is the divisor $m_{1} y_{1}$ $+\cdots+m_{t} y_{t}$ in $Y$. Let $S$ and $D$ be as in (1). Finally, put $\underline{S}=Y^{t} \times S, \underline{X}=Y \times$ $\underline{S}$, and take $\underline{W}$ and $\underline{D}$ to be the pullbacks of $\underline{m} \Delta$ and $D$ to $\underline{X}$. In the present situation, the incidence correspondence of $\underline{W}$ in $\underline{D}$ consists of the points $\left(y_{1}, \ldots, y_{t}, s\right) \in \underline{S}$ such that $D_{s} \geqslant \sum m_{i} y_{i}$ holds. Roughly, the classical formula of de Jonquières counts the number of such points (if finite and provided $t+\operatorname{dim} S$ $\left.=\sum m_{i}\right)$. We retrieve the formula by computing the class of the incidence correspondence in the Chow ring $A(\underline{S})$.

(3) Let $S$ be the Grassmann variety of lines in $\mathbf{P}^{n}$ and let $P \rightarrow S$ be the universal $\mathbf{P}^{1}$-bundle. Put $\underline{S}=P \times_{S} \cdots \times_{S} P$ (t-times) and put $\underline{X}=P \times{ }_{s} \underline{S}$. Now let $\underline{m} \Delta \subset \underline{X}$ denote the sum of multiples $\sum m_{i} \Delta_{0 i}$ of the relative diagonals. Given a hypersurface $H \subset \mathbf{P}^{n}$, set $D=P \cap(H \times S)$, then take $\underline{D}$ to be the pullback of $D$ to $\underline{X}$. The incidence correpondence of $\underline{m} \Delta$ in $\underline{D}$ now consists of the points $\left(y_{1}, \ldots, y_{t}, l\right)$ in $\mathbf{P}^{n} \times \cdots \times \mathbf{P}^{n} \times S$ such that either the line $l$ is contained in $H$ or $H$ cuts out in $l$ a divisor $\geqslant \sum m_{i} y_{i}$. This is certainly an appropriate setup for counting lines satisfying prescribed contact conditions with a hypersurface.

(2.2) Proposition. Let $f: \underline{X} \rightarrow \underline{S}$ be a map of schemes, let $\underline{W} \subset \underline{X}$ be a closed subscheme, and let $\underline{D} \subset \underline{X}$ be the scheme of zeros of a section of an invertible $\vartheta_{X}$-module L. Suppose $f_{*}\left(L \otimes \vartheta_{W}\right)$ is locally free and that its formation commutes with base change (e.g. if $\underline{W}$ is flat and proper $/ \underline{S}$ and $R^{1} \underline{f}_{*}\left(L \otimes \hat{\theta}_{\underline{W}}\right)=0$ holds). Then the incidence correspondence of $\underline{W}$ in $\underline{D}$ exists and is equal to the scheme of zeros of a section of the locally free $\Theta_{\underline{S}}-$ module $\underline{f}_{*}\left(\theta_{\underline{W}} \otimes L\right)$.

Proof. There is a natural diagram of maps of $\theta_{\underline{X}}$-modules,

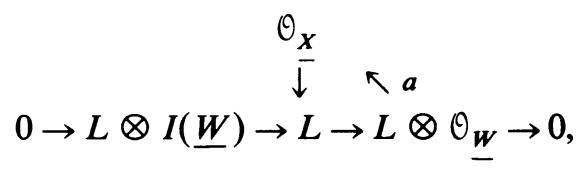

where the vertical arrow is the section defining $\underline{D}$.

Now it is clear that, for each map $T \rightarrow \underline{S}, \underline{D}_{T} \supseteq \underline{W}_{T}$ holds if and only if $a_{T}=0$. The assertion now follows from Altman and Kleiman [1, Proposition (2.3)].

3. Relative dimension 1. In order to apply (2.2) to the preceding examples, we start by working out their main common features.

(3.1) Fix a smooth map $f: X \rightarrow S$. Let $D$ denote the scheme of zeros of a section of an invertible $\theta_{X}$-module $L$. Let $\underline{m}=m_{1}, \ldots, m_{t}$ be a sequence of nonnegative 
integers. Unless stated otherwise, we will assume $t=1$ if the relative dimension of $f$ is $>1$. We denote the $t$-fold cartesian product of $X$ over $S$ by $X^{t}$. The projections from $X^{t}$ onto (resp. omitting) the $i$ th factor will be denoted by $p_{t, i}$ (resp. $p_{t, i}$ ). The diagonal subscheme of $X^{2}$ over $S$ is denoted by $\Delta$ or $\Delta_{X / s}$. We denote by $m \Delta$ the subscheme with ideal $I(m \Delta)=I(\Delta)^{m}$. The pullback of $\Delta$ to $X^{t}$ via the projection onto the $i, j$ factors is denoted by $\Delta_{i j}$.

We are interested in the set of singular points of the fibres of $D \rightarrow S$. Since each fibre $D_{s}$ is (locally) defined by one equation in $X_{s}$, a point is of multiplicity $\geqslant m$ on $D_{s}$ if and only if the local equation of $D_{s}$ lies in the $m$ th power of the maximal ideal there. (If $D_{s}=X_{s}$, each point of $X_{s}$ is considered to be of multiplicity $\geqslant m$ for every $m$.) In order to globalize this observation, as well as to treat the case of several assigned multiplicities, we are lead to consider the subscheme $\underline{m} \Delta \subset X$ $\times_{s} X^{t}$ defined by the ideal

$$
I(\underline{m} D)=I\left(\Delta_{01}\right)^{m_{1}} \ldots I\left(\Delta_{0 t}\right)^{m_{t}} \quad \text { (product). }
$$

(3.2) LeMMA. $\underline{m} \Delta$ is finite and flat over $X^{t}$.

Proof. The subscheme $m \Delta$ of $X^{2}$ is flat over $X$ (via, say, $p_{2,1}$ ). Indeed, this assertion is trivial for $m=1$. For $m \geqslant 2$, we consider the exact sequence

$$
0 \rightarrow I^{m-1} / I^{m} \rightarrow \Theta_{m \Delta} \rightarrow \Theta_{(m-1) \Delta} \rightarrow 0
$$

where $I$ is short for $I(\Delta)$. Because $X$ is smooth $/ S$, each $I^{m-1} / I^{m}$ is a locally free $\theta_{X}$-module (in fact isomorphic to the symmetric power $S_{m-1} \Omega_{X / S}^{1}$ ). Thus $m \Delta$ is flat over $X$ as claimed. For $t \geqslant 2$ (hence rel $\operatorname{dim}=1$ ), the proof is easy and will be omitted.

(3.3) We define the $\underline{m}$-Jacobian of $D$ as the incidence correspondence of $\underline{m} \Delta$ in $p_{t+1,1}^{-1} D$. It exists by (2.2), because $\underline{m} \Delta$ is finite and flat over $X^{t}$. It will be denoted by $J(\underline{m} ; D)$. The $\underline{m}$-contact sheaf of $\bar{L}$ is the $\Theta_{X^{t}}$-module

$$
\mathcal{E}_{X / S}(\underline{m} ; L)=\left(p_{t+1, \hat{i}}\right)\left(\theta_{\underline{m} \Delta} \otimes p_{t+1,1}^{*} L\right) .
$$

(This is a "secant bundle" in the sense of Schwarzenberger [23].) It will also be written simply $\mathcal{E}_{X}(\underline{m} ; L)$ or $\mathscr{E}(\underline{m})$ if no confusion is likely. The $\underline{m}$-contact section is the section of $\mathscr{E}_{X / S}(\underline{m} ; L)$ which is the adjoint (= direct image via $\left.p_{t+1, \hat{i}}\right)$ of the composition

$$
\vartheta^{p_{t+1,1}^{*}} \stackrel{s}{\rightarrow} p_{t+1,1}^{*} L \stackrel{r}{\rightarrow} L \otimes \Theta_{\underline{m} \Delta} .
$$

Here $s$ is the section of $L$ defining $D$ and $r$ is induced by the restriction $\theta \rightarrow \theta_{m \Delta}$. We say $D$ is $\underline{m}$-regular if the Koszul complex of the $\underline{m}$-contact section is exact. If $S$ is Cohen-Macaulay, then $D$ is $\underline{m}$-regular iff $J(\underline{m} ; D)$ is of the right codimension rank $\mathcal{E}(\underline{m})$

$$
\left(=\sum_{i=1}^{t}\left(\begin{array}{c}
m_{i}+n-1 \\
n
\end{array}\right),\right.
$$

where $n=\operatorname{rel} \operatorname{dim} X / S$ (cf. (3.5) below)). 
(3.4) Proposition. (1) $J(\underline{m} ; D)$ is the scheme of zeros of the $\underline{m}$-contact section.

(2) Suppose $D$ is $\underline{m}$-regular. Then $J(\underline{m} ; D)$ repesents the top Chern class of $\mathcal{E}_{X / S}(\underline{m} ; L)$ (in any decent intersection theory).

Proof. (1) The assertion follows from (2.2).

(2) The assertion is well known for nonsingular $S$ [6, p. 153]. For the general case, we sketch a proof in the lemma below, for lack of reference.

(3.4.1) Lemma. Let $X$ be a quasiprojective scheme. Let $\mathcal{E}$ be a locally free $\Theta_{X}$-module of rank $\rho$. Let $s$ be a section of $\mathcal{E}$ and let $Z$ denote the scheme of zeros of $s$. If $s$ is regular (i.e., the Koszul complex $\dot{\Lambda} \mathcal{E}^{*} \rightarrow \vartheta_{Z}$ is exact) then $[Z]=c_{\rho}(\mathscr{E}) \cap$ $[X]$ in $A .(X)$.

Proof. We proceed by induction on the rank of $\mathcal{E}$. When $\rho=1$, then $Z$ is a Cartier divisor, $\mathscr{E}=\mathcal{O}_{X}(Z)$ and the assertion is proven in Fulton [5, p. 160]. Suppose $\rho \geqslant 2$ and consider $p: \mathbf{P}(\mathscr{E}) \rightarrow X$. Now $p^{*}: A .(X) \rightarrow A .(\mathbf{P}(\mathscr{E}))$ is injective, according to Verdier [26, Assertion 6.9, p. 170]. Thus we may assume $\mathscr{E}$ fits into an exact sequence $\mathcal{E}^{\prime} \hookrightarrow \mathcal{E} \rightarrow \mathcal{E}^{\prime \prime}$, where $\mathcal{E}^{\prime \prime}$ is an invertible $\vartheta_{X}$-module. We get a section $s^{\prime \prime}$ of $\mathcal{E}^{\prime \prime}$ which is easily seen to be regular. Denoting by $Z^{\prime \prime}$ the scheme of zeros of $s^{\prime \prime}$, we have $\left[Z^{\prime \prime}\right]=c_{1}\left(\mathcal{E}^{\prime \prime}\right) \cap[X]$. Let $i$ : $Z^{\prime \prime} \subset X$ be the inclusion map. Clearly, $i^{*} s$ factors through a section $s^{\prime}$ of $i^{*} \mathcal{E}^{\prime}$. It can easily be seen that $s^{\prime}$ is also regular and that its scheme of zeros is just $Z$. Hence, we have, by induction,

$$
[Z]=c_{\rho-1}\left(i^{*} \mathcal{E}^{\prime}\right) \cap\left[Z^{\prime \prime}\right] \text { in } A .\left(Z^{\prime \prime}\right) .
$$

We push forward to $A .(X)$ and use the projection formula. Now standard properties of Chern classes yield the assertion.

Next we compute the class of the $\underline{m}$-contact sheaf in the Grothendieck ring $K X^{t}$. We start with the case $t=1$ (and arbitrary relative dimension).

(3.5) Proposition. We have the formula

$$
\varepsilon_{X / S}(m ; L)=L \sum_{0}^{m-1} S_{i}\left(\Omega_{X / S}^{1}\right) \text { in } K X .
$$

( $S_{i}=$ ith symmetric power.) In particular,

$$
\text { rank } \mathcal{E}_{X / S}(m ; L)=\left(\begin{array}{c}
m+n-1 \\
n
\end{array}\right)
$$

where $n=\operatorname{rel} \operatorname{dim} X / S$.

Proof. We argue as in the proof of (3.2).

Tensoring the sequence (3.2.1) by $p_{2,1}^{*} L$ and applying $\left(p_{2, \hat{i}}\right)_{*}$ yields the exact sequence

$$
0 \rightarrow L \otimes S_{m-1}\left(\Omega_{X / S}^{1}\right) \rightarrow \mathcal{E}(m) \rightarrow \mathcal{E}(m-1) \rightarrow 0,
$$

where $\mathscr{E}(m)$ is short for $\mathcal{E}_{X / S}(m ; L)$. The formula now follows by induction on $m$.

(3.5.1) REMARK. $\mathscr{E}(m):=\mathcal{E}_{X / S}(m ; L)$ is equal to EGA's sheaf of principal parts of order $m-1$ [EGA IV $\left.\mathrm{IV}_{4}, 16.7 .1 .2\right]$. 
Here is what $\mathscr{E}(m)$ looks like, locally. Suppose $X=\operatorname{Spec} B, S=\operatorname{Spec} A, L=$ $\vartheta_{X}, \Omega_{B / A}=B d x_{1} \oplus \cdots \oplus B d x_{n}$. Now $\mathcal{E}(m)$ corresponds to the quotient $B \otimes_{A} B / I^{m}$, where $I$ denotes the diagonal ideal of $B \otimes_{A} B$ generated by all $b \otimes 1-1 \otimes b(b$ in $B)$, and the $B$-module structure is induced by $b_{0}\left(b_{1} \otimes b_{2}\right)=$ $\left(b_{0} b_{1}\right) \otimes b_{2}$. Recall that $\Omega_{B / A}=I / I^{2}$ and that the universal $A$-derivation of $B$ is given by $d b=\overline{b \otimes 1-1 \otimes b}\left(\right.$ class $\left.\bmod I^{2}\right) . \mathcal{E}(m)$ splits in the direct sum of the $I^{i} / I^{i+1}(i=0, \ldots, m-1)$, and each of these is free with basis $d x_{1}^{i_{1}} \ldots d x_{n}^{i_{n}}$, where the exponents range over all sequences of nonnegative integers which add up to $i$. Denote the dual basis by $\partial_{i_{1} \ldots i_{n}}$. Then, for each $b$ in $B$, the class of $1 \otimes b$ $\bmod I^{m}$ is equal to

$$
\sum\left(\partial_{i_{1}} \ldots i_{n} b\right) d x_{1}^{i_{1}} \ldots d x_{n}^{i_{n}} .
$$

Let $F$ be a local equation of $D$. Then the $m$-contact section has the coordinates $\partial_{i_{1} \ldots i_{n}} F$. Therefore, $J(m ; D)$ is defined locally by the $\left(\begin{array}{c}m+n-1 \\ n\end{array}\right)$ equations $\partial_{i_{1} \ldots i_{n}} F=$ 0 .

(3.6) THEOREM. We have, in the Grothedieck ring $K^{*}\left(X^{t}\right)$, the formula

$$
\mathcal{E}_{X / S}(\underline{m} ; L)=\sum_{i=1}^{t}\left(p_{t, i}^{*} \mathcal{E}_{X / S}\left(m_{i} ; L\right)\right)\left(-\sum_{h>i} m_{h} \Delta_{h i}\right) .
$$

(The last parenthesis stand for the twisting by the ideal of a divisor.)

Proof. We assume $t \geqslant 2$. Write for short $q_{j}=p_{t+1, j}(j=0, \ldots, t), p_{1}=p_{t, i}$, and similarly for $q_{j}, p_{i}$. Consider the closed subscheme $W$ of $X \times{ }_{s} X^{t}$ with ideal

$$
I(W)=I\left(m_{2} \Delta_{02}\right) \cdots I\left(m_{t} \Delta_{0 t}\right) \quad \text { (products). }
$$

Thus, $I(\underline{m} \Delta)=I\left(m_{1} \Delta_{01}\right) I(W)$ holds. Because $I(W)$ is invertible, we get an exact sequence

$$
0 \rightarrow \hat{\theta}_{m_{1} \Delta_{01}} \otimes I(W) \rightarrow \hat{\theta}_{\underline{m} \Delta} \rightarrow \mathcal{O}_{W} \rightarrow 0 .
$$

Tensoring it with $q_{0}^{*} L$ and applying $\left(q_{\hat{0}}\right)_{*}$ yields the exact sequence

$$
\begin{array}{cc}
0 \rightarrow\left(q_{\hat{0}}\right)_{*}\left(\left(_{m_{1} \Delta_{01}} \otimes I(W) \otimes q_{0}^{*} L\right) \rightarrow \mathcal{E}_{X / S}(\underline{m} ; L) \rightarrow\left(q_{\hat{0}}\right)_{*}\left(\mathcal{O}_{W} \otimes q_{0}^{*} L\right) \rightarrow 0\right. \\
\|(\text { definition }) & p_{1}^{*} \mathcal{E}_{X / S}\left(\underline{m}^{\prime} ; L\right)
\end{array}
$$

where $\underline{m}^{\prime}$ denotes the sequence $m_{2}, \ldots, m_{t}$. The latter equality holds by the Principle of Exchange (or flat base change). Here is the relevant diagram:

$$
\begin{array}{ccc}
W \subset X \times{ }_{S} X^{t} & \stackrel{q_{0}}{\rightarrow} & X^{t} \\
\downarrow q_{1} & & \downarrow p_{\hat{1}} \\
\underline{m}^{\prime} \Delta \subset X \times{ }_{S} X^{t-1} & \stackrel{p_{0}}{\rightarrow} & X^{t-1} \\
\swarrow p_{0} & & \\
X & &
\end{array}
$$


By induction on $t$, it follows that $p_{1}^{*} \delta_{X / S}\left(\underline{m}^{\prime} ; L\right)$ is the sum of the $(t-1)$-last terms of the proposed formula.

Now it remains to identify $A$ in (3.6.1) with $p_{1}^{*} \mathcal{E}_{X / S}\left(m_{1} ; L\right)\left(-\Sigma_{h>1} m_{h} \Delta_{h i}\right)$. For this, we observe that $X \times_{S} X^{t}$ is equal to the fiber product $X^{t} \times{ }_{X^{t-1}} X^{t}$, where we regard $X^{t}$ as a scheme over $X^{t-1}$ via $p_{\hat{\imath}}$. Further, with this identification in mind, the projections onto the 1 st and 2 nd factors are equal to $q_{\hat{0}}$ and $q_{\hat{1}}$ (see diagram). Moreover $\Delta_{01}$ is precisely $\Delta_{X^{t} / X^{t-1}}$. Thus, applying (3.5) to $X^{t} \rightarrow X^{t-1}$ and $p_{1}^{*} L \otimes$ $I(W)$ (in place of $X \rightarrow S$ and $L$, respectively), we get

$$
A=\varepsilon_{X^{t} / X^{t-1}}\left(m_{1} ; p_{1}^{*} L \otimes I(W)\right)=p_{1}^{*} L \otimes I(W) \sum_{0}^{m_{1}-1} S_{i}\left(\Omega_{X^{t} / X^{t-1}}^{1}\right)
$$

Observe that $p_{\hat{1}}: X^{t} \rightarrow X^{t-1}$ is the pullback of $X \rightarrow S:$

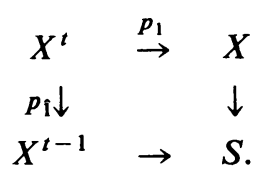

Finally, since formation of relative differentials and of symmetric powers of a locally free module commutes with base change, we see that $A$ is indeed equal to the 1st term of our formula. This completes the proof of the theorem.

The recursive relation stated below will be needed later for the proof of the formula of de Jonquières.

(3.6.2) Proposition. The contact sheaves satisfy the relation

$$
\mathcal{E}_{(\underline{m, u+1}, L)}=\mathcal{E}_{(\underline{m}, u ; L)}+p_{t+1}^{*}\left(L\left(\Omega_{X / S}^{1}\right)^{u}\right)\left(-\sum m_{h} \Delta_{h, t+1}\right) \text { in } K\left(X^{t+1}\right) .
$$

Proof. Consider the fibre square

$$
\begin{array}{ccc}
X \times_{S} X^{t+1} & \stackrel{j}{\hookrightarrow} & X \times_{S} X^{t+2} \\
\uparrow q_{\hat{0}}^{\prime} & & \downarrow q_{\hat{0}} \\
X^{t+1} & \stackrel{i}{\hookrightarrow} & X^{t+2} \\
\left(x_{1}, \ldots, x_{t+1}\right) & \mapsto & \left(x_{t+1}, x_{1}, \ldots, x_{t}, x_{t+1}\right) .
\end{array}
$$

where the horizontal maps are diagonal embeddings. Set $W=(1, \underline{m}, u) \Delta$, that is, the subscheme of $X \times{ }_{s} X^{t+2}$ with ideal $I\left(\Delta_{01}\right) I\left(\Delta_{02}\right)^{m_{1}} \ldots I\left(\Delta_{0 t+1}\right)^{m_{1}} I\left(\Delta_{0, t+2}\right)^{u}$. Since $W$ is flat over $X^{t+2}$, we have the formula

$$
I\left(j^{-1}(W)\right)=j^{*} I(W) .
$$

By the construction of $j$, we have

$$
j^{*} I(W)=I\left(\Delta_{0, t+1}\right)^{u+1} I\left(\Delta_{01}\right)^{m_{1}} \ldots I\left(\Delta_{0 t}\right)^{m_{1}} .
$$


Thus, $j^{-1}(W)$ is just $(\underline{m}, u+1) \Delta$. Consequently, we may write

$$
\begin{aligned}
\mathcal{E}_{X / S}(\underline{m}, u+1 ; L) & =\left(q_{\hat{0}}^{\prime}\right)_{*}\left(\Theta_{(\underline{m}, u+1) \Delta} \otimes\left(q_{0}^{\prime}\right)^{*} L\right) \quad \text { (by definition) } \\
& =\left(q_{\hat{0}}^{\prime}\right)_{*}\left(j^{*}\left(\mathcal{O}_{W} \otimes q_{0}^{*} L\right)\right) \\
& =i^{*}\left(q_{\hat{0}}\right)_{*}\left(\vartheta_{W} \otimes q_{0}^{*} L\right) \quad \text { (by the Principle of Exchange) } \\
& =i^{*} \mathscr{E}_{X / S}(1, \underline{m}, u ; L) \\
& =\left[i^{*} q_{1}^{*} L\left(-u \Delta_{t+2,1}-\sum m_{h} \Delta_{t+2, h+1}\right)\right. \\
& \left.\quad+\sum q_{l+1}^{*} \mathscr{E}_{l}+q_{t+2}^{*} \mathscr{E}_{X / S}(u ; L)\right] \text { in } K^{\cdot}\left(X^{t+1}\right)
\end{aligned}
$$

(by (3.6)), where $\mathcal{E}_{l}$ is short for

$$
\varepsilon_{X / S}\left(m_{l} ; L\right)\left(-u \Delta_{t+2}, l+1-\sum_{h>l} m_{h} \Delta_{h+1}, l+1\right) .
$$

By definition of $i$, we have

$$
q_{l} i= \begin{cases}p_{t+1} & \text { for } l=1 \text { or } t+2 \\ p_{l-1} & \text { for } 2 \leqslant l \leqslant t+1\end{cases}
$$

We also have

$$
i^{*} I\left(\Delta_{h+1, l+1}\right)= \begin{cases}p_{t+1}^{*} \Omega_{X / s}^{1} & \text { for }(h+1, l+1)=(t+2,1), \\ I\left(\Delta_{h, l}\right) & \text { for } 1 \leqslant l<h \leqslant t+1 .\end{cases}
$$

Therefore we get

$$
\begin{aligned}
\mathcal{E}_{X / S}(\underline{m}, u+1 ; L)= & p_{t+1}^{*} L\left(\Omega_{X / S}^{1}\right)^{u}\left(-\sum m_{h} \Delta_{t+1, h}\right) \\
& +\sum p_{l}^{*} \mathcal{E}_{X / S}\left(m_{l} ; L\right)\left(-\sum_{h>l} m_{h}^{\prime} \Delta_{h l}\right),
\end{aligned}
$$

where we put $m_{h}^{\prime}=m_{h}$ for $h \leqslant t$ and $m_{t+1}^{\prime}=u$. By (3.6) the last term in the expression above is precisely $\mathcal{E}(\underline{m}, u ; L)$ in $K^{\cdot}\left(X^{t+1}\right)$, thus completing the proof.

4. Applications. Let $Y, S$ and $D$ be as in (2.1)(1). Let $M$ be an invertible $\mathcal{O}_{Y}$-module associated to the linear system $S$. Put $X=Y \times S$ and $L=M \otimes$ $\vartheta_{S}(1)$. There is a section of $L$ defining the universal divisor $D \subset X$. Now, if the codimension of $J(\underline{m} ; D)$ in $X^{t}$ is equal to the rank of $\mathcal{E}_{X / S}(\underline{m} ; L)$, we have

$$
[J(\underline{m} ; D)]=c_{\text {top }} \mathcal{E}_{X / S}(\underline{m} ; L) .
$$

(4.1) Proposition. Preserve the above notation. If we also assume $J(\underline{m} ; D)$ finite then the degree of its zero cycle is the integer $|\underline{m} ; M|$ defined by

$$
|\underline{m} ; M|=\int c_{t n} \mathcal{E}_{Y}(\underline{m} ; M),
$$

where we put $n=\operatorname{dim} Y$.

Proof. The degree may be computed by first pushing forward the zero cycle from $A\left(X^{t}\right)$ to $A\left(Y^{t}\right)$. Since $X^{t}$ is the trivial projective bundle $Y^{t} \times S$, the assertion 
follows from standard properties of Chern classes and the fact that

$$
\mathcal{E}_{X / S}(\underline{m} ; L)=\mathcal{E}_{Y}(\underline{m} ; M) \otimes \theta_{S}(1)
$$

(4.2) Degree of the dual variety. If $S$ is induced by the hyperplane sections of $Y \subset \mathbf{P}^{N}$, then

$$
|2 ; M|=c_{n} \mathcal{E}_{Y}(2 ; M)=c_{n}\left(M+M \Omega_{Y}^{1}\right)
$$

is the degree of the dual variety of $Y$ (up to the inseparable degree of the dual map $[11$, p. 250]).

(4.3) The Zeuthen-Segre invariant. Suppose $Y$ is a surface. Set $\chi:=c_{2}\left(\Omega_{Y}^{1}\right)$, $K:=c_{1}\left(\Omega_{Y}^{1}\right)$ and (by abuse) $M:=c_{1}(M)$. Suppose $\operatorname{dim} S=1$. Then the formula for $|2 ; M|$ is equivalent to that expressing the classical Zeuthen-Segre invariant $I$ in terms of $\chi$. Indeed, set

$\beta:=$ \# base points,

$\gamma:=$ \# singular members,

$g:=$ (arithmetic) genus of a member of $S$.

Then $I$ is defined classically by

$I:=\gamma-\beta-4 g$ (cf. Baker [2, p. 185]).

On the other hand, we may compute

$$
\begin{aligned}
\gamma & =|2 ; M| \quad(\text { by }(4.1)) \\
& =\chi+2 K M+3 M^{2} \quad(\text { by }(4.2 .1)) \\
& =\chi+2\left(K M+M^{2}\right)+M^{2}=\chi+4(g-1)+\beta .
\end{aligned}
$$

(The assertion $\beta=M^{2}$ holds because the base points are the zeros of the map $\mathcal{O}_{Y}^{\oplus 2} \rightarrow M$ defined by a choice of 2 members of $S$.)

Thus, we retrieve the well-known formula $I+4=\chi$ (cf. Iversen [9, p. 974]).

(4.4) The formula of de Jonquières. Suppose now $Y$ is a smooth projective curve of genus $g$ and $S$ is a linear system associated to an injective $\mathcal{O}_{Y}$-module $M$ of degree $d$. The $\underline{m}$-Jacobian $J(\underline{m} ; D)$ presently parametrizes the points $\left(y_{1}, \ldots, y_{t}, s\right)$ $\in Y^{t} \times S$ such that $D_{s} \geqslant \sum m_{i} y_{i}$. Suppose $t+r=\Sigma m_{i}<d$. If $J(m ; D)$ is finite, then the formula of de Jonquières expresses the degree of its zero cycle. Setting $v=d-\sum m_{i}$, we have

$$
\int[J]=\frac{\left(\Pi m_{i}\right)}{v !} \sum_{h=0}^{t}(t+v-h) ! h !\left(\begin{array}{l}
g \\
h
\end{array}\right) \sigma_{h}\left(m_{1}-1, \ldots, m_{t}-1\right),
$$

where $\sigma_{0}=1$, and $\sigma_{1}, \ldots, \sigma_{t}$ denote the elementary symmetric functions in $t$ variables.

Recent proofs of the formula used some deep numerical relations in the Jacobian of $Y$ (cf. Mattuck [19]). The rest of this paragraph is devoted to the proof of (4.4.1).

By (4.1), we have $\int[J]=|\underline{m} ; M|$. Denote by $[\underline{m}]$ the r.h.s. of (4.4.1). We claim that

$$
|\underline{m} ; M|=[\underline{m}] \text {. }
$$

To prove the latter equality (and hence (4.4.1)), we will need the following. 
(4.4.3) LEMMA. There are formulas:

(1) $|\underline{m}, 0 ; M|=0$,

(2) $|\underline{m}, 1 ; M|=\left(d-\sum m_{i}\right)|\underline{m} ; M|$,

(3) $|1, \ldots, 1 ; M|=(d !) /(d-t)$ !,

(4)

$$
\begin{aligned}
|\underline{m}, u+1 ; M|= & |\underline{m}, u ; M|+(d+u(2 g-2))|\underline{m} ; M(-u y)| \\
& -\sum m_{i}\left|m_{1}, \ldots, m_{i}+u, \ldots, m_{t} ; M\right|,
\end{aligned}
$$

where $y$ is a point of $Y$ and $M(-u y):=M \otimes I(y)^{u}$.

(5) $|\underline{m} ; M|=\pi \underline{m} ; M \mid$, where $\pi \underline{m}$ denote a permutation of the $m_{i}$.

Proof of (4.4.2). Replacing $\underline{m}$ by $\underline{m}, 1, \ldots, 1$, we may assume $v=0$, because of (4.4.3)(2). If $\underline{m}=1, \ldots, 1$ ( $t$ times), then $[\underline{m}]=t$ !. This is just fine by (4.4.3)(3), because $d=\bar{t}$ (as $v=0)$. Now it is clear that (4.4.3)(3)-(4.4.3)(5) completely determine $|\underline{m} ; M|$. So, to finish the proof, it suffices to verify that $[\underline{m}]$ satisfies the following recursive relation:

$$
\begin{aligned}
{[\underline{m}, u+1]=} & {[\underline{m}, u, 1]+(d+u(2 g-2))[\underline{m}, 1] } \\
& -\sum m_{i}\left[m_{1}, \ldots, m_{i}+u, \ldots, m_{t}, 1\right]
\end{aligned}
$$

with $1+u+\sum m_{i}=d$. The verification offers no surprises and will be omitted.

Proof of (4.4.3). Clearly, (2) implies (3). Also, (2) is a consequence of (1) and (4).

To prove (5), let $\pi_{0}$ denote the permutation of the coordinates of $Y \times Y^{t}$ such that $p_{0} \pi_{0}=p_{0}$ and $p_{i} \pi_{0}=p_{\pi i}$ for $i=1, \ldots, t$. We clearly have that $\pi_{0}\left(\Delta_{01}\right)=\Delta_{0, \pi i}$, and therefore, $\pi_{0}^{-1}(\underline{m} \Delta)=(\pi \underline{m}) \Delta$, where $\pi \underline{m}:=m_{\pi 1}, \ldots, m_{\pi t}$. With a slight abuse of notation we may write, by definition of contact sheaves,

$$
\begin{aligned}
\pi^{*} \mathcal{E}_{Y}(\underline{m} ; M) & =\pi^{*}\left(p_{\hat{0}}\right)_{*}\left(p_{0}^{*} M \otimes \vartheta_{\underline{m} \Delta}\right)=\left(p_{\hat{0}}\right)_{*} \pi_{0}^{*}\left(p_{0}^{*} M \otimes \vartheta_{\underline{m} \Delta}\right) \\
& =\left(p_{\hat{0}}\right)_{*}\left(p_{0}^{*} M \otimes \pi_{0}^{*} \vartheta_{\underline{m} \Delta}\right)=\mathcal{E}_{Y}(\pi \underline{m} ; M) .
\end{aligned}
$$

As $\pi^{*}=\pi_{*}^{-1}$ holds (because $\pi$ is an automorphism of $Y^{t}$ ), we get

$$
\int c_{t} \mathcal{E}_{Y}(\underline{m} ; M)=\int \pi_{*} \pi^{*} c_{t} \mathcal{E}_{Y}(\underline{m} ; M)=\int \pi_{*} c_{t} \mathcal{E}_{Y}(\pi \underline{m} ; M)=\int c_{t} \mathcal{E}_{Y}(\pi \underline{m} ; M),
$$

thus proving (5).

For the remaining assertions, set, for short, $\mathcal{E}_{(u)}:=\mathcal{E}_{Y}(\underline{m}, u ; M)$. To prove (1), notice that $\mathcal{E}(0)=p^{*} \mathcal{E}_{Y}(\underline{m} ; M)$, where $p: Y^{t+1} \rightarrow Y^{t}$ omits the last coordinate. (1) now follows because the r.h.s. of this last equality has zero $(t+1)$-Chern class.

To prove (4), we recall from (3.6.2) the following formula:

$$
\mathcal{E}(u+1)=\mathcal{E}(u)+\left(q_{t+1}^{*}\left(M \Omega_{Y}^{\otimes u}\right)\right)\left(-\sum_{h<t} m_{h} \Delta_{h, t+1}\right) .
$$

Let $y$ and $\underline{h}$ denote, respectively, the embeddings of $Y^{t}$ onto $Y^{t} \times y$, and $\Delta_{h, t+1}$ in $Y^{t+\overline{1}}$. We compute Chern classes modulo algebraic equivalence. Thus $c_{1}\left(M \Omega_{Y}^{\otimes u}\right)=$ $(d+u(2 g-2)) y$. Using the above formula to compute $c_{t+1} \mathcal{E}(u+1)$, we get

$$
|\underline{m}, u+1 ; M|=|\underline{m}, u ; M|+(d+u(2 g-2)) \int c_{t} \underline{y}^{*} \mathcal{E}(u)-\sum m_{h} \int c_{t} \underline{h}^{*} \mathcal{E}(u) .
$$


Using (3.6) together with the formulas

$$
\begin{gathered}
\left(q_{t+1} \underline{y}\right)\left(Y^{t}\right)=\{y\}, \quad \underline{y}^{*}\left(\Delta_{t+1, i}\right)=q_{i}^{*}(y), \\
\underline{y}^{*}\left(\Delta_{h, i}\right)=\Delta_{h, i} \quad(i<h \leqslant t), \quad q_{i} \underline{y}=q_{i} \quad(1 \leqslant i \leqslant t),
\end{gathered}
$$

we get

$$
\underline{y}^{*} \mathcal{E}(u)=\mathcal{O}_{Y^{\prime}}^{\oplus u}+\sum q_{i}^{*} \mathcal{E}_{Y}\left(m_{i} ; M(-u y)\right)\left(-\sum_{h>i} m_{h} \Delta_{h i}\right) \text { in } K\left(Y^{t}\right) .
$$

Hence,

$$
\int c_{t} \underline{y}^{*} \mathcal{E}(u)=|\underline{m} ; M(-u y)| .
$$

Similarly, we get, for $h=t$,

$$
\begin{aligned}
\underline{t}^{*} \mathcal{E}(u)= & q_{t}^{*} \mathcal{E}_{Y}(u ; M)+q_{t}^{*} \mathcal{E}_{Y}\left(m_{t} ; M\right) \Omega_{Y}^{\otimes u} \\
& +\sum_{1}^{t-1} q_{i}^{*} \mathcal{E}_{Y}\left(m_{i} ; M\right)\left(-u \Delta_{t, i}-m_{t} \Delta_{t, i}-\sum_{i+1}^{t-1} m_{j} \Delta_{j i}\right) .
\end{aligned}
$$

However, the 1st two terms in the r.h.s. adds up precisely to

$$
q_{t}^{*} \mathcal{E}_{Y}\left(u+m_{t} ; M\right) ! ! !
$$

Hence, we have proven

$$
\underline{t}^{*} \mathcal{E}(u)=\mathcal{E}_{Y}\left(m_{1}, \ldots, m_{t-1}, m_{t}+u ; M\right) .
$$

Finally, with the help of permutations on $Y^{t}$ and $Y^{t+1}$ (cf. proof of (5)) we get, for any $h=1, \ldots, t$,

$$
\underline{h}^{*} \mathcal{E}(u)=\mathcal{E}_{Y}\left(m_{1}, \ldots, m_{h}+u, \ldots, m_{t} ; M\right),
$$

thus completing the proof of (4.4.3).

5. Lines with prescribed contact with a hypersurface. Fix a projective space $\mathbf{P}^{r}=\mathbf{P}(V)$. Let $S$ denote the Grassmann variety of lines in $\mathbf{P}^{r}$. Let $Q$ denote the universal 2-quotient of $V_{s}$. Thus $X:=\mathrm{P}(Q) \rightarrow S$ is the universal family of lines. Let $H \subset \mathbf{P}^{r}$ be a hypersurface of degree $d$, and let $s_{H} \in H^{0}\left(\mathbf{P}^{r}, \mathcal{O}(d)\right)$ be a defining equation. Now $s_{H}$ gives rise to a section of $L:=\vartheta_{X}(d)$ whose scheme of zeros $D \subset X$ is just the intersection $X \cap(H \times S) \subset \mathbf{P}^{r} \times S$. We say a line $l$ and the hypersurface $H$ have $\underline{m}$-contact with each other if $l \cap H$ contains a divisor of $l$ of the form $\sum m_{i} x_{i}$. The scheme $J(\underline{m} ; D)$ presently parametrizes the pairs $\left(\left(x_{1}, \ldots, x_{t}\right), l\right) \in H \times \cdots \times H \times S$ such that $l \cap H \geqslant \sum m_{i} x_{i}$. Thus, one may retrieve classical formulas for the number of lines satisfying prescribed contact conditions with a hypersurface by computing the appropriate $c . \mathcal{E}_{X / S}\left(\underline{m} ; \theta_{X}(d)\right)$. Let us make a few explicit calculations for a surface in $\mathbf{P}^{3}$.

Set $\lambda=c_{1}(Q)$ and set $\pi=c_{2} Q$. Thus, $\lambda$ (resp. $\pi$ ) is the class of the "condition" that a line meet a fixed other line (resp. that a line pass through a fixed point). There are formulas:

$$
\begin{aligned}
& \lambda^{4}=2(=\# \text { of lines meeting } 4 \text { others }) \\
& \pi^{2}=1 \text { (= \# of lines through } 2 \text { points ...), } \\
& \lambda^{2} \pi=1 \text { (= \# of lines meeting } 2 \text { others and passing through a point). }
\end{aligned}
$$


Set $\theta=c_{1} \theta_{X}(1)$. We need the Chern class of $\Omega:=\Omega_{X / s}^{1}$. In view of the canonical sequence on $X=\mathbf{P}(Q), 0 \rightarrow \Omega(1) \rightarrow Q_{X} \rightarrow \vartheta_{X}(1) \rightarrow 0$, we can compute $w:=c_{1} \Omega$ as $w=\lambda-2 \theta$.

We also have the formulas $\theta^{2}=\lambda \theta-\pi, \theta^{3}=\left(\lambda^{2}-\pi\right) \theta-\lambda \pi, \theta^{4}=0$. (The 1st holds by Grothendieck's construction of Chern classes; the 2 nd then follows.)

(5.1) Five-fold contact with a surface of degree $d(t=1, m=5)$. The number we are after is the degree of the zero cycle

$$
J(5 ; D)=c_{5} \mathcal{E}_{X / S}\left(5 ; \vartheta_{X}(d)\right) .
$$

By (3.5), this is the term of top degree in $(1+d \theta)(1+d \theta+w) \ldots(1+d \theta+4 w)$. Using, as needed, the relations listed above, and pushing down to $S$, one finds the zero cycle

$$
d\left[24 \lambda^{4}+(50 d-192) \lambda^{4}+\left(35 d^{2}-300 d+576\right)\left(\lambda^{4}-\pi \lambda^{2}\right)\right]
$$

which has degree $5 d\left(7 d^{2}-40 d+48\right)$ (cf. Baker [2, Formula (4), p. 90]).

(5.2) Doubly inflexional lines $\left(t=2 ; m_{1}=m_{2}=3\right)$. Their number is one-half the degree of the zero cycle

$$
\begin{aligned}
z:= & c_{6} \mathcal{E}_{X / S}\left(3,3 ; \Theta_{X}(d)\right)=\left(d \theta_{1}-3 \Delta\right)\left[(d-2) \theta_{1}-3 \Delta+\lambda\right] \\
& \cdot\left[(d-4) \theta_{1}-3 \Delta+2 \lambda\right] \cdot d \theta_{2}\left[(d-2) \theta_{2}+\lambda\right]\left[(d-4) \theta_{2}+2 \lambda\right]
\end{aligned}
$$

(The indices mean pullback to $X \times_{S} X$ via the corresponding projection.)

Using the formulas $\Delta^{2}=-c_{1}(\Omega)=-w \Delta=(2 \theta-\lambda) \Delta, p_{2 *}(\Delta)=p_{2 *}\left(\theta_{1}\right)=1$ and $p_{2 *}(1)=0$, we may compute $p_{2 *}(z)$. We retrieve the number

$$
d(d-4)(d-5)\left(d^{3}+3 d^{2}+29 d-60\right)
$$

(cf. Baker [2, Formula (5), p. 91], or Schubert [22, Formula (19), p. 236]).

(5.3) Tritangent lines $\left(t=3, m_{1}=m_{2}=m_{3}=2\right)$ meeting a general line. One has to calculate the degree of

$$
z:=\lambda c_{6} \delta\left(2,2,2 ; \vartheta_{X}(d)\right)=\lambda \prod_{i=1}^{3}\left(d \theta_{i}-2 \sum_{i}\right)\left((d-2) \theta_{i}+\lambda-2 \sum_{i}\right),
$$

where $\Sigma_{i}:=\sum_{h>i} \Delta_{h i}$. We get 6 times the number

$$
\frac{1}{3} d(d-3)(d-4)(d-5)\left(d^{2}+3 d-2\right)
$$

(cf. Schubert [22, Formula (15), p. 236]).

6. Arbitrary relative dimension. Let $Y$ be a smooth variety and let $D \subset Y$ be a positive divisor. We say that $D$ has a singularity of type $\underline{m}$ if the following holds: first require that $D$ have a point $y_{1}$ of multiplicity $>m_{1}$; next let $Y_{1}$ denote the blowup to $Y$ at $y_{1}$, let $E_{1}$ denote the exceptional divisor and let $D_{1}$ denote the total transform of $D$; then require that the effective divisor $D_{1}-m_{1} E_{1}$ have a point $y_{2}$ of multiplicity $\geqslant m_{2}$, and so on. The sequence $\left(y_{1}, y_{2}, \ldots\right)$ thus constructed is called a singularity of type $\underline{m}$ of $D$.

Of course, if $\operatorname{dim} Y=\overline{1}$, it is clear that $D$ has a singularity of type $\underline{m}$ iff $D \geqslant \sum m_{i} y_{i}$ holds.

(6.1) Let $f: X \rightarrow S, D \subset X$ and $L$ be as in (3.1). We no longer impose restrictions on the relative dimension. Our goal is to construct an $S$-scheme $J(\underline{m} ; D)$ whose 
fibre over any point $s$ in $S$ consists of the sequences of singularities of type $\underline{m}$ of the fibre $D_{s}$.

We first define inductively a sequence of maps

$$
p_{t, 1}, p_{t, 2}: X\{t\} \rightarrow X\{t-1\}
$$

We set $X\{0\}:=S, X\{1\}:=X$ and $p_{1,1}=p_{1,2}:=f$. For $t \geqslant 2$, we view $X\{t-1\}$ as a scheme $/ X\{t-2\}$ via $p_{t-1,1}$, and we let

$$
b_{t}: X\{t\} \rightarrow X\{t-1\} \times_{X\{t-2\}} X\{t-1\}
$$

denote the blowup of the diagonal. Then we set $p_{t, i}=p_{i} b_{t}$, where $p_{i}$ denotes the projection onto $X\{t-1\}$. Note that $p_{t, i}$ is smooth [EGA IV $_{4}, 19.4 .8$ ]. We denote the exceptional divisor by $E_{t}$ (or $E$ if no confusion is likely) and the $m$ th power of its deal by $\theta_{t}(m)$.

For each $\theta_{X}$-module $\mathcal{Q}$, we set

$$
\mathbb{Q}\left(m_{1}\right):=\mathcal{O}_{2}\left(m_{1}\right) \otimes p_{2,2}^{*} \mathbb{Q}
$$

and define inductively, for $t \geqslant 2$,

$$
\mathcal{Q}(\underline{m}):=\mathcal{O}_{t+1}\left(m_{t}\right) \otimes p_{t+1,2}^{*} \mathfrak{A}\left(m_{1}, \ldots, m_{t-1}\right) .
$$

$J(\underline{m} ; D)$ will be a subscheme of $X\{t\}$, defined by the following step-by-step procedure.

For $t=1, m=m_{1}$, the subscheme $J(m ; D) \subset X$ was already constructed in (3.3). The result below will enable us to go on assigning more singular points, one at a time.

(6.2) Proposition. $J(m ; D)$ is also equal to the incidence correspondence of $m E$ in $p_{2,2}^{-1} D$.

$$
\begin{gathered}
m E \subset X\{2\} \supset p_{2,2}^{-1} D \\
p_{2,1} \swarrow \quad \searrow p_{2,2} \\
J(m ; D) \subset X \quad X \supset D
\end{gathered}
$$

Proof. The diagram (2.2.1) now reads

$$
0 \rightarrow L(m) \rightarrow p_{2,2}^{*} L \rightarrow p_{2,2}^{*} L^{\star} \otimes \vartheta_{m E} \rightarrow 0 .
$$

Using the formulas

$$
\begin{gathered}
\left(R^{i} p_{2,1}\right)_{*} L(m)=\left(R^{i} p_{1}\right)_{*}\left(p_{2}^{*} L \otimes I(\Delta)^{m}\right), \\
\left(R^{i} p_{2,1}\right)_{*}\left(p_{2,2}^{*} L \otimes \vartheta_{m E}\right)=\left(R^{i} p_{1}\right)_{*}\left(p_{2}^{*} L \otimes \vartheta_{m \Delta}\right)
\end{gathered}
$$

(see Manin [18, p. 63]), one shows that $m E$ is flat $/ X$, that $\left(R^{1} p_{2,1}\right)_{*}\left(p_{2,2}^{*} L \otimes \mathcal{O}_{m E}\right)$ $=0$ and that the adjoint of $a$ in $X$ coincides with the $m$-contact section. This proves the proposition.

(6.3) Continuing with our program of constructing $J(\underline{m} ; D)$, notice that, because $m E$ is flat $X$, the sequence (6.2.1) remains exact after restriction over $J:=$ $J(m ; D)$. Hence, the section of $p_{2,2}^{*} L$ that defines $p_{2,2}^{-1} D$ yields a section $s_{m}$ of 
$L(m)_{J}$. We call the scheme of zeros of $s_{m}$ the m-virtual transform of $D$, and denote it by $D(m)$.

At this stage, we replace $D \subset X \rightarrow S$ by $D(m) \subset X\{2\}_{J} \rightarrow J$ and define, by induction,

$$
J(\underline{m} ; D):=J\left(m_{2}, \ldots, m_{t} ; D\left(m_{1}\right)\right) .
$$

We leave to the reader the verification that (6.3.1) agrees with (3.3).

7. The generic class of $J(\underline{m} ; D)$. Preserve the notation of $\S 6$. Denote by $\underline{m}_{i}$ the truncated sequence $m_{1}, \ldots, m_{i}$.

(7.1) We say $D$ is $\underline{m}$-regular if, for each $i=1, \ldots, t$, the Koszul complex of the $m_{i}$-contact section defining $J\left(\underline{m}_{i} ; D\right)$ in $J\left(\underline{m}_{i-1} ; D\right) \times_{X\{i-1\}} X\{i\}$ is exact.

If $S$ is Cohen-Macaulay, then $D$ is $\underline{m}$-regular iff $\operatorname{each} J\left(\underline{m}_{i} ; D\right)$ is either empty or of the right codimension

$$
\sum_{j=1}^{i}\left(\begin{array}{c}
m_{j}+n-1 \\
n
\end{array}\right)
$$

in $X\{i\}$.

We define inductively the $\underline{m}$-contact virtual sheaf of $L$ by

$$
\mathcal{E}_{X / S}(\underline{m} ; L):=\mathcal{E}_{X\{t\} / X\{t-1\}}\left(m_{t} ; L\left(\underline{m}_{t}\right)\right)+p_{t, 1}^{*} \mathcal{E}_{X / S}\left(\underline{m}_{t} ; L\right) \quad \text { in } K^{\cdot}(X\{t\}),
$$

where the first term on the r.h.s. is the (class of the) $m_{t}$-contact sheaf of $L\left(\underline{m}_{t}\right)$ (6.1.1).

(7.2) Lemma. We have the formula, in $K^{\cdot}(X\{t\})$,

$$
\mathcal{E}_{X / S}(\underline{m} ; L)=\mathcal{E}_{X\{2\} / X}\left(m_{2}, \ldots, m_{t} ; L\left(m_{1}\right)\right)+\left(p_{t, 1}^{*} \ldots p_{2,1}^{*}\right) \mathcal{E}_{X / S}\left(m_{1} ; L\right) .
$$

Proof. The assertion follows immediately from the definitions. Just observe that, replacing $X \rightarrow S$ by $\tilde{X}:=X\{2\} \rightarrow \tilde{S}:=X$, we have $\tilde{X}\{t-1\}=X\{t\}$.

(7.3) Theorem. Suppose $D$ is $\underline{m-r e g u l a r . ~ T h e n ~ t h e ~ c l a s s ~ o f ~} J(\underline{m} ; D)$ in $A .(X\{t\})$ is the Poincare dual of the top Chern class of the $\underline{m}$-contact sheaf of $L$. In symbols,

$$
[J(\underline{m} ; D)]=c_{\text {top }}\left(\mathcal{E}_{X / s}(\underline{m} ; L)\right) \cap[X\{t\}] .
$$

Proof. We have, to start with, the equality

$$
\left[J\left(m_{1} ; D\right)\right]=c_{\text {top }}\left(\mathcal{E}_{X / S}\left(m_{1} ; L\right)\right) \cap[X] \text { in } A .(x),
$$

in view of (3.4)(2).

Set $\underline{m}^{\prime}=m_{2}, \ldots, m_{t}$, replace $X \rightarrow S$ by

$$
X^{\prime}:=X\{2\} \times_{X} J\left(m_{1} ; D\right) \rightarrow S^{\prime}:=J\left(m_{1} ; D\right),
$$

$D$ by $D^{\prime}:=D\left(m_{1}\right)(6.3)$, and $L$ by $L^{\prime}:=L\left(m_{1}\right)_{X^{\prime}}$. By construction, we have $J(\underline{m} ; D)=J\left(\underline{m}^{\prime} ; D\right)$. By induction, the class of the latter in $A .\left(X^{\prime}\{t-1\}\right)$ is dual to the top Chern class of $\mathcal{E}_{X^{\prime} / S^{\prime}}\left(\underline{m}^{\prime} ; L^{\prime}\right)$. Now $X^{\prime}\{t-1\}$ is obviously equal to $X\{t\} \times_{X} S^{\prime}$. Since $X\{t\}$ is smooth (hence flat) $X$, the operations of pulling back the homology class of a subscheme and taking the homology class of the pullback 
of that subscheme are interchangeable. Thus, setting, for short, $p=p_{2,1} \cdots p_{t, 1}$, we may write, in $A .(X\{t\})$,

$$
\begin{aligned}
{\left[X^{\prime}\{t-1\}\right] } & =p^{*}\left[J\left(m_{1} ; D\right)\right] \\
& =p^{*} c_{\text {top }}\left(\mathcal{E}_{X / S}\left(m_{1} ; L\right)\right) \cap[X\{\tau\}] .
\end{aligned}
$$

Now, denoting by $i$ the inclusion $X^{\prime}\{t-1\} \subset X\{t\}$, we have

$$
\begin{aligned}
{[J(\underline{m} ; \mathbf{D})] } & =i_{*}\left[J\left(\underline{m}^{\prime} ; \mathbf{D}\left(m_{1}\right)\right)\right] \\
& =i_{*}\left(c_{\text {top }} \mathcal{E}_{X^{\prime} / S^{\prime}}\left(\underline{m}^{\prime}: L^{\prime}\right) \cap\left[X^{\prime}\{t-1\}\right]\right) \quad \text { (introduction) } \\
& =c_{\text {top }} \mathcal{E}_{X\{2\} / X}\left(\underline{m}^{\prime} ; L\left(m_{1}\right)\right) \cap i_{*}\left[X^{\prime}\{t-1\}\right] \quad \text { (projection formula) } \\
& =c_{\text {top }}\left(\mathcal{E}_{X\{2\} / X}\left(\underline{m}^{\prime}: L\left(m_{1}\right)\right)+p^{*} \mathcal{E}_{X / S}\left(m_{1} ; L\right)\right) \cap[X\{t\}] \\
& =c_{\text {top }}\left(\mathcal{E}_{X / S}(\underline{m} ; L)\right) \cap[X\{t\}] \quad(\text { by }(7.2)) . \quad \square
\end{aligned}
$$

(7.4) REMARK. It would be interesting to find explicitly the universal polynomial $p\left(m_{1}, \ldots, m_{t} ; c_{1}, \ldots, c_{n} ; \lambda\right)$ expressing the push down of $c_{\text {top }}\left(\mathcal{E}_{X / S}(\underline{m} ; L)\right) \cap$ $[X\{t\}]$ to $A .(X)$ in terms of $\underline{m}$ and of the Chern classes of $\Omega_{X / s}^{1}$ and $L$. De Jonquières did it for $X$ a curve. For $t=1$ and $X$ a curve or a surface, A. Lascoux related $p(m ; c . ; \lambda)$ to Thom polynomials [15]. We will retrieve several classical formulas of Enumerative Geometry by computing the approrpiate $p(\underline{m} ; c ., \lambda)$

8. More examples. Return to the notation of $\S 4$. We restate (4.1).

(8.1) Proposition. Let $Y$ be a smooth projective variety of dimension $n$. Let $S$ be a linear system associated to an invertible $\theta_{Y}$-module $M$. Let $D$ denote its universal divisor. Suppose $D$ is $\underline{m}$-regular. If

$$
\operatorname{dim} S+t n=\sum\left(\begin{array}{c}
m_{i}+n-1 \\
n
\end{array}\right)
$$

then $J(\underline{m} ; D)$ is finite and the degree $|\underline{m} ; M|$ of its zero cycle is $|\underline{m} ; M|=$ $\int c_{t n} \delta_{Y}(\underline{m} ; M)$.

Proof. See the proof of (4.1).

(8.1.1) Problem. Is $|\underline{m} ; M|=|\pi \underline{m} ; M|$ for $n>1$ ? (See (4.4.3)(5).)

In the first two examples below we take $Y$ to be a surface. We freely use the notation of (4.3).

(8.2) Bitangent planes of a surface in $\mathbf{P}^{3}$. Assume $\operatorname{dim} S=2$. Then the formula for $\int[J(2,2 ; D)]$ is $\left[\int\left(\chi+2 K M+3 M^{2}\right)\right]^{2}-\int\left(7 \chi+6 K^{2}+39 K M+42 M^{2}\right)$. This is derived from (8.1) and (3.5) by pushing down $c_{4} \mathcal{E}_{Y}(2,2 ; M)$ from $A(Y\{2\})$ to $A(Y \times Y)$ and then to $A(Y)$. The main ingredients of the computation are:

(a) The formula

$$
\Omega_{Y\{2\} / Y}^{1}=p_{2,2}^{*} \Omega^{1} \otimes \vartheta(E)+\vartheta(-E)-\vartheta \quad \text { in } K(Y\{2\})
$$

for the class of the cotangent sheaf of $Y\{2\} / Y$ (via $p_{2,1}$, we recall). This formula follows from the two standard exact sequences,

$$
b_{2}^{*} \Omega_{Y \times Y / Y}^{1} \subset \Omega_{Y\{2\} / Y}^{1} \rightarrow \Omega_{Y\{2\} / Y \times Y}^{1}=j_{*} \Omega_{E / \Delta}^{1},
$$


and

$$
\Omega_{E / \Delta} \subset \Omega_{Y}(-1) \rightarrow \theta_{E},
$$

where $j: E \hookrightarrow Y\{2\}$ is the inclusion of the exceptional divisor. (It is worth recalling the well-known facts of a blowup such as $b_{2}$ : first, $E=\mathbf{P}\left(\Omega_{Y}^{1}\right)$; second, the tautological ample sheaf $\vartheta_{E}(1)$ on $E$ is equal to $j^{*} \vartheta_{Y\{2\}}(-E)$.)

(b) The intersection relations

$$
E^{i}=j_{*}\left((-e)^{i-1}\right) \quad \text { in } A(Y\{2\})
$$

where we put $e:=c_{1} \mathfrak{O}_{E}(1)$. We also have $e^{2}=K e-\chi,\left(b_{2} j\right)_{*}(1)=0$ and $\left(b_{2} j\right)_{*}(e)$ $=1_{A(Y)}$.

In particular, if $Y$ is a surface in $\mathbf{P}^{3}$ of degree $d$ and $S$ is a net of plane sections, we have

$$
\begin{aligned}
\int \chi & =\left(d^{2}-4 d+6\right) d \\
\int K^{2} & =(d-4)^{2} d ; \quad \int M^{2}=d ; \quad \int K M=d(d-4) .
\end{aligned}
$$

Substituting these in the formula above, we get

$$
d(d-1)(d-2)\left(d^{3}-d^{2}+d-12\right)
$$

the expression which is twice the number of bitangent planes through a general point (cf. Baker [2, p. 153]).

If $Y$ is the projective plane and $S$ is a net of curves of degree $d$, the number $|2,2 ; M|$ is $9 d^{4}-36 d^{3}+12 d^{2}+81 d-66$. This is twice the degree of the " . . conditions nécessaires pour qu'une courbe plane puisse avoir deux points doubles" (cf. S. Roberts [20, p. 276]).

(8.3) Tritangent planes of a surface in $\mathbf{P}^{3}$. Assume $\operatorname{dim} S=3$. A rather lengthy calculation yields the following expression for $|2,2,2 ; M|$ :

$$
\begin{gathered}
{\left[\int\left(\chi+2 K M+3 M^{2}\right)\right]^{3}-14\left[\int\left(\chi+2 K M+3 M^{2}\right)\right]^{2}} \\
-\int\left(\chi+2 K M+3 M^{2}\right) \int\left(7 \chi+18 K^{2}+84 M^{2}+89 K M\right) \\
+\int\left(138 \chi+376 K^{2}+1380 M^{2}+1576 K M\right) .
\end{gathered}
$$

If $Y$ is $\mathbf{P}^{2}$ and $S$ is a general web of cubics, so that we have $\int \chi=3$, $\int M^{2}=\int K^{2}=9, \int M K=-9$, the formula gives the number $6 \cdot 15$. One can check directly that 15 is precisely the number of triangles containing 6 general points.

If $Y$ is a surface in $\mathbf{P}^{3}$ of degree $d$ and $S$ is the complete system of plane sections, substituting in the values for $\chi, K^{2}$, etc. computed in (8.2.1), we get

$$
d^{9}-6 d^{8}+15 d^{7}-59 d^{6}+204 d^{5}-339 d^{4}+770 d^{3}-2056 d^{2}+1920 d,
$$

which is 6 times the classical formula for the number of tritangent planes (cf. Salmon [21, Formula (vi), p. 292]). We recall that the factor $6=3$ ! comes in 
because $J(2,2,2 ; D)$ accounts for the divisor together with an ordered triplet of singular points. It can be checked that most surfaces of degree $>3$ possess exactly the prescribed number of tritangent planes, though this does not follow from our general criterion (see (9.1) below).

(8.4) Contacts of higher-dimensional linear spaces with a hypersurface. The difficulties with the explicit computations of formulas increase rapidly. Conceptually, however, this is a special case of the situation for divisors with specified singularities on a smooth family. One takes $X \rightarrow S$ to be the universal family of $r$-subspaces in a fixed projective $n$-space $\mathbf{P}^{n}$, or its restriction to a suitable subvariety of the Grassmannian of $r$-spaces in $\mathbf{P}^{n}$. Each hypersurface $H$ in $\mathbf{P}^{n}$ of degree $d$ induces a subscheme $D_{H} \subset X$, given by the zeros of a section of $L:=\vartheta_{X}(d)$. The fibre of $D_{H}$ over [ $r]$ in $S$ is the intersection $H \cap[r]$. In this situation, we can again play the game of successive blowing-ups and compute the generic class of $J\left(\underline{m} ; D_{H}\right)$.

The reader is invited to carry out the computations leading to the formula below.

(8.4.1) Number of 2-planes in $\mathbf{P}^{4}$ meeting a line and cutting a general hypersurface of degree $d \geqslant 4$ in a curve with one triple point and another double point:

$$
20 d^{7}-161 d^{6}+510 d^{5}-1,617 d^{4}+6,176 d^{3}-12,916 d^{2}+10,080 d .
$$

That is, the degree of the zero cycle $c_{9}\left(\mathcal{O}_{X / S}\left(3,2 ; \theta_{X}(d)\right)\right) \sigma$, where $X / S$ is the universal family of 2-planes in $\mathbf{P}^{4}$ and $\sigma$ denotes the special Schubert cycle of 2-planes meeting a line.

(8.5) Degree of the condition that a surface in $\mathbf{P}^{3}$ acquire 2 double points. Take $Y:=\mathbf{P}^{3}, M:=\mathcal{O}_{\mathbf{P}^{3}}(d), S:=$ complete system of surfaces of degree $d$. For $d \geqslant 3$ we know $(9.1)(3) D$ is $(2,2)$-regular. The subvariety of $S$ parametrizing the surfaces with (at least) 2 (possibly infinitely near) double points is integral and of codimension 2. Its degree is, for char $k \neq 2$,

$$
\frac{1}{2} \int c_{6} \mathcal{E}_{Y}(2,2 ; M)=2(d-1)^{2}(d-2)\left(4 d^{3}-8 d^{2}+8 d-25\right),
$$

in agreement with S. Roberts [20, top of p. 277].

(8.6) Curves with specified coincidences of tangents at a singularity. Let $f: X \rightarrow S$ be proper and smooth of relative dimension 2. Let $D \subset X$ be the scheme of zeros of a section of an invertible $\theta_{X}$-module $L$.

We have defined, for each positive integer $m$, a closed subscheme $J:=J(m ; D)$ of $X$, which parametrizes the points $x$ of $X$ such that the fibre $D_{s}$ of $D$ over $s=f(x)$ contains $x$ as an $m$-fold point. "In general", there should be $m$ distinct tangent directions to $D_{s}$ at $x$. Now, for each sequence $\underline{n}:=n_{1}, \ldots, n_{t}$ of positive integers such that $n_{1}+\cdots+n_{t} \leqslant m$, one may ask for the generic homology class of the set of $x$ for which there are tangent directions $\tau_{1}, \ldots, \tau_{t}$ at $x$ (in the surface $X_{s}$ ) such that $n_{1}$ of the tangents to $D_{s}$ at $x$ coincide with $\tau_{1}, n_{2}$ with $\tau_{2}$, etc. We discuss here the general setup, then "compute" the generic homology class we sought, and finally compute it explicitly in a few cases.

Consider the $m$-virtual (projectivized) tangent cone of $D$, i.e., the intersection $T_{m} D=D(m) \cap E \subset X\{2\}$ of the $m$-virtual transform of $D(6.3)$ with the exceptional divisor $E$. Thus, $T_{m} D$ is the scheme of zeros of the restriction of the section 
$s_{m}$ of $L(m)_{J}(6.3)$ to $C:=E_{J}$. Notice that, for each $x$ in $J$, the fibre $E_{x}$ is the projective line of tangent directions of the surface $X_{s}$ at $x$ (where $s=f(x)$ ). If $x$ is $m$-fold, not $(m+1)$-fold in $D_{s}$, then $\left(T_{m} D\right)_{x}$ is the positive divisor of the tangent directions of $D_{s}$ at $x$. If $x$ is $(m+1)$-fold in $D_{s}$ then $\left(T_{m} D\right)_{x}=E_{s}$.

So we are back to the happy situation where we have got a family of curves (in fact projective lines) $C \rightarrow J$, together with the scheme of zeros of an invertible ${ }{ }_{C}$-module. We call $J\left(\underline{n} ; T_{m} D\right)$ the scheme of tangent coincidences of type $n$ of $D$. We say $D$ is $(m ; n)$-regular if $D$ is $m$-regular and $T_{m} D$ is $\underline{n}$-regular.

Applying (7.3) twice, we get, whenever $D$ is $(m ; n)$-regular,

$$
\left[J\left(\underline{n} ; T_{m} D\right)\right]=c_{\nu}\left(\mathcal{E}_{X / S}(m ; L)+\mathcal{E}_{E / X}\left(\underline{n} ; L(m)_{E}\right)\right) \text { in } A .\left(E^{t}\right),
$$

where $\nu:=\left(\begin{array}{c}m+1 \\ 2\end{array}\right)+\sum n_{i}$.

Suppose now $D$ is the universal divisor of a linear system $S$ on a smooth, projective surface $Y$ associated to the invertible $\theta_{Y}$-module $M$. Set $\mathbf{P}=\mathbf{P}\left(\Omega_{Y}^{1}\right)$. Suppose

$$
\operatorname{dim} S=\left(\begin{array}{c}
m+1 \\
2
\end{array}\right)-2-t+\sum_{1}^{t} n_{i}
$$

If $D$ is $(m ; n)$-regular then $J\left(\underline{n} ; T_{m} D\right)$ is finite and the degree of its zero cycle is the degree of

$$
c_{t+2}\left(\mathcal{E}_{Y}(m ; M)+M \mathcal{E}_{\mathbf{P} / Y}\left(\underline{n} ; \mathcal{\theta}_{P}(m)\right)\right) .
$$

This follows from (8.6.1), recalling that now $L=M \otimes \theta_{S}(1), X=Y \times S$ and $E=\mathbf{P} \times S$.

(8.6.3) Cusps of a general net on a surface. Taking $m=2$ and $\underline{n}=2$ in the above formula and pushing down to $A(Y)$, we find $2\left(6 M^{2}+6 M K+K^{2}+\chi\right)$ (cf. Lascoux [15, p. 151]).

(8.6.4) Cuspnodes of a general web on a surface. We start with our surface $Y$ and a general web $S$ (= 3-dimensional linear system). Construct $Z:=J(2 ; D) \subset X=S$ $\times Y$. Then look at the restriction $B \rightarrow Z$ of $X\{2\}=S \times Y\{2\}$ over $Z$, together with the 2-virtual transform $D^{\prime}$ of the universal divisor of $S$. Finally, take $T:=$ $J\left(2 ; T_{2} D^{\prime}\right)$. This gives us the cuspidal points of the fibres of $D^{\prime}$ over $Z$, which is what we were after. Computing the class of $T$ and pushing it down from $S \times \mathbf{P}$ to $\mathbf{P}:=\mathbf{P}\left(\Omega_{Y\{2\} / Y}^{1}\right)$, then to $Y\{2\}$, then to $Y \times Y$ and finally to $Y$, we find, for the number of cuspnodes, the formula

$$
\begin{aligned}
2\left\{\left[\int\left(\chi+2 K M+3 M^{2}\right)\right]^{2}+\int\left(K^{2}+\right.\right. & \left.4 K M+3 M^{2}\right) \int\left(\chi+2 K M+3 M^{2}\right) \\
& \left.-\int\left(12 \chi+36 K^{2}+144 K M+120 M^{2}\right)\right\} .
\end{aligned}
$$

Computing for $Y=\mathbf{P}^{2}$ and $M=\hat{\theta}_{\mathbf{P}^{2}}(3)$ one finds zero. This can be checked directly by analyzing the possible degenerations of a cubic. The only ones with a double point and a cusp are the unions of double lines with another line. But these form a family of dimension 4 , which can, therefore, be safely avoided by a general web of plane cubics. 
Computing for $Y=$ surface in $\mathrm{P}^{3}$ of degree $d$ and $M=\theta_{Y}(1)$, subsituting in the values for $\chi$, etc. from (8.2.1), we get, lo and behold, precisely the number $4 d(d-2)(d-3)\left(d^{3}+3 d-16\right)$ of Salmon [21, Formula (4), p. 292].

9. Conditions for $m$-regularity. Fix a smooth, surjective and proper map $g: Y \rightarrow Z$ and let $M$ be an invertible $\vartheta_{Y}$-module. Suppose $V:=g_{*} M$ is locally free. Set $S:=\mathbf{P}\left(V^{*}\right)$ and $X:=S \times_{Z} Y=\mathbf{P}\left(V_{Y}^{*}\right)$. Let $f: X \rightarrow S$ be the pullback of $g$. The natural map of $\theta_{Y}$-modules $V_{Y} \rightarrow M$ gives rise to a section $s$ of $L:=\mathcal{\theta}_{X}(1) \otimes M$ just as in $\S 4$. Again we denote by $D$ the scheme of zeros of $s$ in $X$ and call it the universal divisor of $M$.

We perform the constructions of (6.1) with $Y \rightarrow Z$ in place of $X \rightarrow S$. Denote by $q_{t, 1}, q_{t, 2}$ the maps $Y\{t\} \rightarrow Y\{t-1\}$ of (6.1). Clearly, the maps $p_{t, i}: X\{t\} \rightarrow$ $X\{t-1\}$ are just the pullbacks of $q_{t, i}$, and each $X\{t\}$ is now the projective bundle $\mathbf{P}\left(V_{Y(t)}^{*}\right)$.

For the statement of the theorem, we recall (6.1.1) that $M(m)$ stands for the successive twistings of $M$ by powers of the ideals of the exceptional divisors. Thus, $M\left(m_{1}\right)=\left(q_{2,2}^{*} M\right) \otimes \mathcal{O}_{2}\left(m_{1}\right)$, etc $\ldots$

(9.1) Theorem. (1) Suppose $\left(R^{1} q_{t+1,1}\right)_{*} M(\underline{m})=0$. Then $J(\underline{m} ; D)$ is a projective $Y\{t\}$-subbundle of $X\{t\}=\mathbf{P}\left(V_{Y(t)}^{*}\right)$ of the right codimension

$$
\left(=\sum\left(\begin{array}{c}
m_{i}+n-1 \\
n
\end{array}\right), n:=\operatorname{rel} \operatorname{dim} Y / Z\right)
$$

and $D$ is $\underline{m}$-regular.

(2) $\left(R^{1} q_{t+1,1}\right)_{*} M(\underline{m})=0$ provided that either

(a) $\underline{m}$ satisfies the relaxed proximity inequalities

$$
m_{i-1} \geqslant m_{i}+\cdots+m_{t}-1 \text { for } i=2, \ldots, t,
$$

and $M$ is a sufficiently high multiple of some $\theta_{Y}$-module $N$ ample $/ Z$; or

(b) the relative dimension of $Y / Z$ is 1 and

$$
\text { degree } M_{Y(z)} \geqslant 2(\text { genus } Y(z))-2+\sum m_{i}
$$

for all $z$ in $Z$.

(3) If $Y \rightarrow Z$ is a projective bundle (e.g. $Z=\operatorname{Spec} k, Y=\mathbf{P}^{n}$ ) and $M$ is the rth power of the canonical ample line bundle then $D$ is $\underline{m}$ regular provided $r>\left(\sum m_{i}\right)-1$ holds and $\underline{m}$ satisfies (9.1.1).

(9.2) Lemma. Suppose $\left(R^{1} q_{2,1}\right)_{*} M(m)=0$ for some positive integer $m$. Then:

(1) $R^{1} g_{*} M=0$;

(2) $V:=g_{*} M$ and $V_{m}:=\left(q_{2,1}\right)_{*} M(m)$ are locally free and there is a canonical exact sequence

$$
0 \rightarrow V_{m} \rightarrow g^{*} V \rightarrow \mathcal{E}_{Y / Z}(m ; M) \rightarrow 0
$$

such that the dual surjection $g^{*} V^{*} \rightarrow V_{m}^{*}$ yields the identification

$$
J(m ; D)=\mathbf{P}\left(V_{m}^{*}\right) \subset \mathbf{P}\left(g^{*} V^{*}\right)=X ;
$$

(3) the m-virtual transform (6.3) of the universal divisor of $M$ is equal to the universal divisor of $M(m)$. 
Grant the lemma. We proceed to prove (1) of the theorem. Set $\underline{m}_{i}:=m_{1}, \ldots, m_{i-1}$. Applying (1) of the lemma to $q_{t, 1}, M\left(\underline{m}_{t}\right)$ and $m_{t}$ in place of $g$, $M$ and $m$, we get that $\left(R^{1} q_{t, 1}\right)_{*} M\left(\underline{m}_{t}\right)=0$. Repeating the process, we get down to $\left(R^{1} q_{2,1}\right)_{*} M\left(m_{1}\right)=0$. Hence $J\left(m_{1} ; D\right)$ and $\mathbf{P}\left(V_{m_{1}}^{*}\right)$ are equal. Now replace $g, M$ and $\underline{m}$ by $q_{2,1}, M\left(m_{1}\right)$ and $m_{2}, \ldots, m_{t}$. We have proven, by induction on $t$, that $\bar{J}(\underline{m} ; D)$ is equal to the $Y\{t\}$-projective subbundle $\mathbf{P}\left(V_{\underline{m}}^{*}\right) \subset X\{t\}$, where $V_{\underline{m}}:=\left(q_{t+1,1}\right)_{*} M(\underline{m})$ is locally free and fits into the exact sequence

$$
0 \rightarrow V_{\underline{m}} \rightarrow q_{t, 1}^{*} V_{\underline{m}} \rightarrow \mathcal{E}_{Y\{t\} / Y\{t-1\}}\left(m_{t} ; M\left(\underline{m}_{t}\right)\right) \rightarrow 0 .
$$

This completes the proof of (1) of the theorem.

Proof of Lemma (9.2). We apply $\left(q_{2,1}\right)_{*}$ to the canonical exact sequence

$$
0 \rightarrow M(m) \rightarrow q_{2,2}^{*} M \rightarrow q_{2,2}^{*} M \otimes \vartheta_{m E} \rightarrow 0 .
$$

We get the exact sequence (9.2.1), together with the formula

$$
\left(R^{1} q_{2,1}\right)_{*} q_{2,2}^{*} M=0 \text {. }
$$

However, the left-hand side is equal to $\left(R^{1} q_{2}\right)_{*} q_{1}^{*} M$. By flat base change, the latter is just $g^{*}\left(R^{1} g_{*} M\right)$. Since $g$ is faithfully flat, we get $R^{1} g_{*} M=0$.

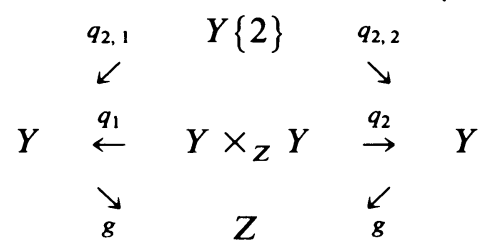

Twisting (9.2.1) by $\Theta_{\mathbf{P}\left(V^{*}\right)}(1)$ we get the diagram of $\Theta_{X}$-modules

$$
\begin{gathered}
\vartheta_{X} \\
\downarrow \quad v_{m} \\
0 \rightarrow V_{m}(1) \rightarrow V_{X}(1) \rightarrow \varepsilon_{X / S}(m ; L) \rightarrow 0 .
\end{gathered}
$$

Here the vertical arrow arises from the universal quotient $u: V_{X}^{*} \rightarrow \vartheta_{X}(1)$ by dualizing and twisting. The slant arrow $s$ is just the $m$-contact section (3.3). It follows that, for any map $T \rightarrow X, s_{T}=0$ holds iff $u_{T}$ factors through $V_{T}^{*} \rightarrow\left(V_{m}^{*}\right)_{T}$. This proves (2) of the lemma. The remaining assertion follows from the definition (6.3) together with the fact that the formation of $g_{*} M$ commutes with base change.

The main ingredient of the proof of (2) of the theorem is the following.

(9.3) Lemma (OF PROXIMITY INEQUALITIES). Fix a regular scheme $B$. Set $B_{1}:=B$, and for $i \geqslant 2$ let $b_{i}: B_{i}+B_{i-1}$ denote the blowup of $B_{i-1}$ at a point $y_{i-1}$. Set $b:=b_{2} \ldots b_{t+1}$. For each $\Theta_{B_{t+1}}$-module $\mathbb{Q}$, set

$$
\mathbb{Q}(\underline{m}):=\mathcal{O}_{B_{t+1}}\left(m_{t}\right) \otimes b_{t+1}^{*} \mathcal{O}_{B_{t}}\left(m_{t-1}\right) \otimes \cdots \otimes b^{*} \vartheta_{B_{2}}\left(m_{1}\right) \otimes \mathbb{Q} .
$$

Suppose $\underline{m}$ satisfies the inequalities (9.2.1). Then $\left(R^{1} b\right)_{*} \vartheta(\underline{m})=0$.

Proof. The lemma is an immediate consequence of the following assertion. Let $\Re_{j}$ denote the ideal of a point $z_{j}$ in $B_{t+1}$. For each sequence of nonnegative integers $\underline{n}:=n_{1}, \ldots, n_{s}$, set $\mathfrak{T}^{\underline{n}}:=\mathscr{T}_{1}^{n_{1}} \ldots \mathfrak{T}_{s}^{n_{s}}$ (product of ideals). Then

$$
\left(R^{1} b\right)_{*}\left(\mathscr{T}^{\underline{n}}(\underline{m})\right)=0
$$


provided the new sequences $m_{1}, \ldots, m_{t}, n_{1}+\cdots+n_{s}$ satisfy the inequalities (9.1.1).

We proceed for the proof of (9.3.1) by induction on $t$. Suppose $t=1$. For each $j$ such that $z_{j}$ is not in the exceptional divisior $E:=b^{-1}\left(y_{1}\right)$, we have

$$
R^{i} b_{*}\left(\Re^{n}(m)\right)=\Re_{j}^{n_{j}} R^{i} b_{*}\left(\Re^{n}(m)\right)
$$

where $\underline{n}_{j}:=n_{1}, \ldots, n_{j-1}, 0, n_{j+1}, \ldots, n_{s}$. Thus, we may assume each $z_{j}$ lies in $E$. Without loss of generality, we may assume the $z_{j}$ are distinct. Now, there is an exact sequence,

$$
0 \rightarrow \Re^{n-1}(1) \rightarrow \Re^{\underline{n}} \rightarrow \Re^{\underline{n}} \Theta_{E} \rightarrow 0
$$

where 1 denotes the sequence equal to 1 on each slot where $n_{j}$ is $>0$ and zero otherwise. Tensoring it with $\Theta_{B_{2}}(m)$ and applying $b_{*}$, we get the exact sequence

$$
R^{1} b_{*}\left(\Re^{-\underline{n}-1}(m+1)\right) \rightarrow R^{1} b_{*}\left(\Re^{n}(m)\right) \rightarrow R^{1} b_{*}\left(\Re^{n} \Theta_{E}(m)\right) .
$$

By induction on $\max \left\{n_{1}, \ldots, n_{s}\right\}$, the 1 st term is zero. The last term is just $H^{1}\left(E, \Re\left(\underline{n} \vartheta_{E}(m)\right)\right.$, which is zero for $m \geqslant n_{1}+\cdots+n_{s}-1$ because $E$ is a projective space. This finishes the proof for $t=1$.

Assume $t \geqslant 2$. Set $b^{\prime}:=b_{2} \ldots b_{t}$. Thus, $b=b^{\prime} b_{t+1}$. We study the exact sequence (derived from the spectral sequence of composite functors)

$$
R^{1} b_{*}^{\prime}\left(b_{t+1}\right)_{*}(A) \rightarrow R^{1} b_{*}(A) \rightarrow b_{*}^{\prime}\left[\left(R^{1} b_{t+1}\right)_{*} A\right],
$$

where we set for short $A:=\mathfrak{N}^{\underline{n}}(\underline{m})$. By the projection formula, we have

$$
\left(R^{1} b_{t+1}\right)_{*}(A)=\Theta\left(\underline{m}_{t}\right)\left(R^{1} b_{t+1}\right)_{*}\left(\Re^{n}\left(m_{t}\right)\right) \text {. }
$$

This is zero by the case $t=1$. Now reorder the $z_{j}$ so that $z_{j}$ is in $E_{t+1}$ iff $r \leqslant j \leqslant s$. Set $\underline{n}^{\prime}=n_{1}, \ldots, n_{r-1}$ and $\underline{n}^{\prime \prime}=n_{r}, \ldots, n_{s}$. We have the exact sequence,

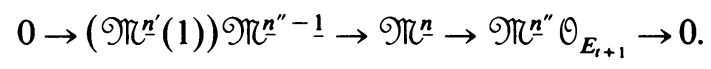

Twist it by $\Theta_{B_{t+1}}\left(m_{t}\right)$ and apply $\left(b_{t+1}\right)_{*}$. By the case $t=1$, we get a short exact sequence of direct images. Twisting this by $\mathcal{O}\left(\underline{m}_{t}\right)$ and applying $b_{*}^{\prime}$ finally yields the exact sequence

$$
\begin{aligned}
R^{1} b_{*}^{\prime}\left(\Theta\left(\underline{m}_{t}\right) b_{t+1 *}\right. & \left.\left(\left(\mathscr{N} \underline{n}^{\prime}\left(m_{t}+1\right)\right) \Re^{\underline{n}^{\prime \prime}-1}\right)\right) \\
& \rightarrow R^{1} b_{*}^{\prime}\left(\Theta\left(\underline{m}_{t}\right) b_{t+1^{*}}\left(\left(\mathscr{N} \underline{\underline{n}}^{\prime}\left(m_{t}\right)\right) \mathfrak{N}^{\underline{n}^{\prime \prime}}\right)\right) \rightarrow 0 .
\end{aligned}
$$

The last zero is right because $\left(b_{t+1}\right)_{*}\left(\mathscr{N}^{\underline{n}} \underline{\underline{n}}^{\prime \prime} \Theta_{E_{t+1}}\right)$ is supported at one point. Arguing by induction on $\max \left(\underline{n}^{\prime \prime}\right)$, we deduce that the middle term is also zero, thus completing the proof of (9.3).

Proof of (2) OF THE THEOREM. Let $y$ be a point of $Y\{t\}$, and let $y_{i}$ denote its image in $Y\{i\}$ (via $q_{i+1,1} \ldots q_{t+1,1}$ ). Let $B_{i}$ denote the fibre of $Y\{i\}$ over $y_{i-1}$. Thus, we have the sequence of blowings-up

$$
B_{t+1} \stackrel{b_{t+1}}{\rightarrow} B_{t} \stackrel{b_{t}}{\rightarrow} \ldots \stackrel{b_{2}}{\rightarrow} B_{1}=Y_{y_{0}}
$$

where $B_{1}$ is the fibre of $Y \rightarrow Z$ containing $y_{1}$ and $b_{i+1}$ is the blowup of $B_{i}$ at $y_{i}$. Set $b:=b_{2} \ldots b_{t+1}$ and set $N_{1}:=N_{B_{1}}$. 
By the Principle of Exchange, it suffices to prove $H^{1}\left(B_{t+1}, N_{1}^{\otimes \nu}(\underline{m})\right)=0$ holds for $\nu \gg 0$. For this, we use the exact sequence, derived from Leray's spectral sequence,

$$
H^{1}\left(B_{1}, N_{1}^{\otimes \nu} \otimes b_{*} \Theta(\underline{m})\right) \rightarrow H^{1}\left(B_{t+1}, N_{1}^{\otimes \nu}(\underline{m})\right) \rightarrow H^{0}\left(B_{1}, N_{1}^{\otimes \nu} \otimes R^{1} b_{*} \vartheta(\underline{m})\right) .
$$

By Lemma (9.3), the last term vanishes (for all $\nu$ ). On the other hand, by ampleness, the 1 st term is zero for $\nu \gg 0$. This proves (2)(a). If $\operatorname{rel} \operatorname{dim} Y / Z$ is 1 , then $B_{1}$ is a curve, each $b_{i}$ is the identity map and $\theta(\underline{m})$ can be identified with $\vartheta_{B_{1}}\left(-m_{1} y_{1}-\cdots-m_{t} y_{t}\right)$. This proves (2)(b).

Proof of (3) OF THE THEOREM. Preserving the notation of the proof of (2), we now have $B_{1}=\mathbf{P}^{n}$ for some $n$, and $N_{1}=\vartheta_{\mathbf{P}^{n}}(1)$. It can be shown that $b_{*} \vartheta(\underline{m})$ contains an $\mathcal{O}_{B_{1}}$-module $A$ of the form $A=\mathfrak{N}_{1}^{n_{1}} \ldots \mathfrak{N}_{s}^{n_{3}}$, where each $\mathfrak{N}_{i}$ is the ideal of a point $P_{i}$ and $\sum n_{i} \leqslant \sum m_{i}$ and $\operatorname{supp}\left(b_{*} \theta(\underline{m}) / A\right)$ is finite. Therefore, $H^{1}\left(B_{1}, N_{1}^{r} b_{*} \Theta(m)\right)$ vanishes provided $H^{1}\left(\mathbf{P}^{n}, A(r)\right)=0$. The latter holds iff the natural map $H^{0}\left(\mathbf{P}^{n}, \vartheta_{\mathbf{P}^{n}}(r)\right) \rightarrow H^{0}\left(\mathbf{P}^{n}, \Theta / A\right)$ is surjective. The surjectivity amounts to the possibility of finding nonhomogeneous polynomials of degree $<r$ in $n$ variables with arbitrarily given Taylor developments around $P_{i}$ up to order $n_{i}-1$, which can be achieved for $r \geqslant-1+\sum n_{i}$.

Problem (1) Suppose $Y$ is the normalization of a plane curve. Consider the linear system cut out by the plane curves of degree $n$. When is its universal divisor m-regular?

(2) Get rid of the artificial condition (9.1.1).

10. Conditions for multiplicity 1 . Let $Y$ be a smooth projective variety of dimension $n$. Let $S$ be an $r$-dimensional linear system on $Y$. Set for short $J:=$ $J(\underline{m} ; D)(D=$ universal divisor of $S)$. Set

$$
j:=r+t n-\sum\left(\begin{array}{c}
m_{i}+n-1 \\
n
\end{array}\right) .
$$

Let $G$ denote the Grassmann variety of codimension $j$ linear subsystems of $S$. We have the following.

(10.1) TheOREM. Suppose $J$ is integral and of the right dimension $j$. Then there exist integers $s$, e such that, for every subsystem $S^{\prime}$ in an open dense subset of $G, J \times{ }_{S} S^{\prime}$ has precisely $s$ distinct points and the multiplicity at each is 1 if $J / S$ is separable and is $(\text { char } k)^{e}$ otherwise.

Proof. We use a variation of the theorem on the transversality of a general translate (cf. Kleiman [14]). Consider the diagram

$$
\begin{array}{ccc}
\bar{J} & \rightarrow J \\
q \swarrow \quad & \downarrow \bar{p} & \downarrow p \\
G \leftarrow \bar{S} & \rightarrow S,
\end{array}
$$

where $\bar{S} \rightarrow G$ is the universal family and the square is cartesian. Since $J$ is integral and $\bar{S} \rightarrow S$ is smooth with integral fibres, therefore $\bar{J}$ is integral. By construction, $q^{-1}\left(S^{\prime}\right)=J \times{ }_{S} S^{\prime}$ for each $S^{\prime}$ in $G$. Also, $\operatorname{dim} \bar{J}=\operatorname{dim} G$. Moreover, $p$ is everywhere ramified iff $\bar{p}$ is. Hence, by the lemma on p. 109 of [22], there are integers $s$ 
and $e$ such that, for each $S^{\prime}$ in some open dense subset of $G, q^{-1}\left(S^{\prime}\right)$ consists of $s$ distinct points and the multiplicity (= length of the artinian local ring of the fibre) at each is 1 or (char $k)^{e}$, as asserted.

It remains to produce some manageable criterion for $J / S$ to be separable. In one direction, if $S$ comes from the hyperplane sections of an embedding $Y \subset \mathbf{P}^{r}$, then $J(2 ; D) \rightarrow S$ is the dual map of Katz [11]. He shows that, possibly after replacing the given embedding by its composition with a Veronese embedding, the dual map is generically unramified iff char $k \neq 2$ or $\operatorname{dim} Y$ is even. On the other hand, we learned from D. Laksov [secret notes] the following result. We no longer restrict $S$ to be a projective space.

(10.2) Proposition. Suppose the relative dimension of $X / S$ is 1 . Then the ramification locus of $J(m ; D) / S$ is equal to $J(m+1 ; D)$ if char $k \nmid m$ and is $J(m ; D)$ if char $k \mid m$.

Proof. We may assume $X=\operatorname{Spec} B, S=\operatorname{Spec} A, \quad L=\mathcal{O}_{X}$ and $\Omega_{B / A}^{1}=B d x$ for some $x$ in $B$. Let $F$ be a local equation of $D$. As explained in (3.5.1), there are differential operators $\partial_{i}: B \rightarrow B$ such that $J:=J(m ; D)$ is cut out by the $m$ equations $\partial_{i} F=0(i=0, \ldots, m-1)$. Furthermore, we have $\partial_{0} b=b$ and $d b=$ $\left(\partial_{1} b\right) d x$ for all $b$ in $B$, and $\partial_{1} \partial_{i}=(i+1) \partial_{i}$ ([EGA IV I $\left.\left._{4}, 16.11 .2 .2\right]\right)$. Recalling the standard exact sequence [EGA IV $\left.\mathrm{I}_{4}, 16.4 .21 .1\right] N_{J / X} \rightarrow\left(\Omega_{X / S}^{1}\right)_{J} \rightarrow \Omega_{J / S}^{1} \rightarrow 0$, we see that the ramification locus of $J / S$ is cut out in $J$ by the equations $\partial_{1} \partial_{i} F=0$ $(i=0, \ldots, m-1)$. These are equivalent to $\partial_{i} F=0(i=0, \ldots, m-1)$ together with $m \partial_{m} F=0$, whence the assertion is proved.

(10.3) CoRollary. (1) If char $k \mid m_{1} \ldots m_{t}$ then the ramification locus of $J(\underline{m} ; D) / S$ is $J(\underline{m} ; D)$. (2) If char $k \nmid m_{1} \ldots m_{t}$ then that ramification locus is contained in the union of the closed subsets $J\left(m_{1}, \ldots, m_{i}+1, \ldots, m_{t} ; D\right)$ and the diagonals.

Proof. The map $J(\underline{m} ; D) \rightarrow S$ factors as

$$
J(\underline{m} ; D) \stackrel{f_{t}}{\rightarrow} \ldots \rightarrow J\left(m_{2} ; D\left(m_{1}\right)\right) \stackrel{f_{2}}{\rightarrow} J\left(m_{1} ; D\right) \stackrel{f_{1}}{\rightarrow} S .
$$

Now, arguing as in the proof of (4.4.3)(5), if $\pi$ denotes a permutation of $\{1, \ldots, t\}$, then $J(\underline{m} ; D)$ is isomorphic to $J(\pi \underline{m} ; D)$ over $S$, where $\pi \underline{m}:=m_{\pi 1}, \ldots, m_{\pi t}$. Thus, if char $k \mid m_{i}$ for some $i$, we may as well assume $i=t$. By the proposition, $f_{t}$ is everywhere ramified, whence so is $f_{1} \ldots f_{t}$.

To prove the 2nd assertion, let $x$ be a point of $J(\underline{m} ; D)$ off the above-mentioned union. Thus, $x=\left(x_{1}, \ldots, x_{t}\right)$, where the $x_{i}$ are distinct points of $X$ lying over the same point $s$ in $S$, with $D_{s}=\Sigma_{1}^{t} m_{h} x_{h}+D^{\prime}$, for some $D^{\prime}>0$ and no $x_{i} \in D^{\prime}$. Consequently, each $f_{i}$ unramified at $\left(x_{1}, \ldots, x_{i}\right)$, whence $f_{1} \ldots f_{t}$ is unramified at $x$.

(10.4) Problem. Conics in char 2 have just one tangent line through a general point; smooth plane cubics have, in general, just 3 inflexional tangent lines. Is the number of bitangent lines to a general quartic in char 2 equal to $14=(28 / 2)$ or 7 ? A similar question applies to example (5.1) in char 5. Is there a general rule for deciding the value of the exponent $e$ in (10.1)? 


\section{REFERENCES}

1. A. Altman and S. L. Kleiman, Foundations of the theory of Fano schemes, Compositio Math. 34 (1977), 3-47.

2. H. F. Baker, Principles of geometry. Vol. VI, Cambridge Univ. Press, Cambridge, 1933.

3. F. Enriques and O. Chisini, Teoria geometrica delle equazioni e delle funzioni algebriche. Vol. II, Nicola Zanichelli Editore, Bologna, 1918.

4. F. Enriques, Le superficie algebriche, Nicola Zanichelli Editore, Bologna, 1949.

5. W. Fulton, Rational equivalence for singular varieties, appendix to Riemann-Roch for singular varieties by P. Baum, W. Fulton and R. MacPherson, Inst. Hautes Etudes Sci. Publ. Math. No. 45, 1975.

6. A. Grothendieck, La théorie des classes de Chern, Bull. Soc. Math. France 86 (1958), 137-159.

7. A. Grothendieck and J. Dieudonné, Éléments de géométrie algébrique (cited [EGA IV $_{4}$ ]), Inst. Hautes Etudes Sci. Publ. Math. No. 32, 1967.

8. D. Hilbert, Mathematical problems, translated by M. W. Newson, Bull. Amer. Math. Soc. 8 (1901-02), 437-479.

9. B. Iversen, Numerical invariants and multiple planes, Amer. J. Math. 92 (1970), 968-996.

10. J. P. E. F. de Jonquières, Mémoire sur les contacts multiples . . , Crelle J. 66 (1866), 289-321.

11. N. Katz, Etude cohomologique des pinceaux de Lefschetz, Groupes de Monodromie en Géométrie Algébrique (SGA VII), Lecture Notes in Math, vol. 340, Springer-Verlag, Berlin and New York, 1970.

12. S. L. Kleiman, Problem 15. Rigorous foundation of Schubert's enumerative calculus, Proc. Sympos. Pure Math., vol. 28, Amer. Math. Soc., Providence, R. I., 1974.

13. The enumerative theory of singularities, Real and Complex Singularities (Oslo, 1976), P. Holm, Editor, Sijthoff \& Noordhoff, Amsterdam, 1977.

14. ___ The transversality of a general translate, Compositio Math. 28 (1974), 287-297.

15. A. Lascoux, Sistemi lineari di divisori sulle curve e sulle superficie, Ann. Mat. Pura Appl. 114 (1977), 141-154.

16. I. MacDonald, Some enumerative formulae for algebraic curves, Proc. Cambridge Philos. Soc. 54 (1958), 399-416.

17. __ Symmetric products of an algebraic curve, Topology 1 (1962), 319-343.

18. Y. I. Manin, Lectures on the K-functor in algebraic geometry, Russian Math. Surveys 24 (1969), $1-89$.

19. A. Mattuck, Secant bundles on symmetric products, Amer. J. Math. 87 (1965), 779-797.

20. S. Roberts, Sur l'ordre des conditions ..., Crelle J. 67 (1867), 266-278.

21. G. Salmon, $A$ treatise on the analytic geometry of three dimensions, 5th ed., Vol. II, Longmans, Green and Co., London, 1915.

22. H. Schubert, Kalkul der abzahlenden Geometrie, Springer-Verlag, Berlin and New York, 1979 (reprint).

23. R. L. E. Schwarzenberger, The secant bundle of a projective variety, Proc. London Math. Soc. 14 (1964), 257-268.

24. C. Segre, Introduzione alla geometria sopra un ente algebrico semplicemente infinito, Ann. Mat. 22 (1894), 41-142.

25. I. Vainsencher, Conics in characteristic 2, Compositio Math. 36 (1978), 101-112.

26. J. L. Verdier, Le théorème de Riemann-Roch pour les variétés algébriques éventuellement singulières (Sém. Bourbaki, Exposé No. 464), Lecture Notes in Math., vol. 514, Springer-Verlag, Berlin and New York, 1976, pp. 159-175.

27. H. G. Zeuthen and M. Pieri, Géométrie énumérative, Encyclopédie des Sciences Mathématiques, Vol. III, 2, Teubner, Leipzig, 1915, pp. 260-331.

Departamento de Matemática, Universidade Federal de Pernambuco, Recife-Pe. Brazil 